Louisiana State University

LSU Digital Commons

2008

\title{
A critical item analysis of the QABF: development of a short form assessment instrument
}

Ashvind Nand Singh

Louisiana State University and Agricultural and Mechanical College

Follow this and additional works at: https://digitalcommons.Isu.edu/gradschool_dissertations

Part of the Psychology Commons

\section{Recommended Citation}

Singh, Ashvind Nand, "A critical item analysis of the QABF: development of a short form assessment instrument" (2008). LSU Doctoral Dissertations. 2624.

https://digitalcommons.Isu.edu/gradschool_dissertations/2624

This Dissertation is brought to you for free and open access by the Graduate School at LSU Digital Commons. It has been accepted for inclusion in LSU Doctoral Dissertations by an authorized graduate school editor of LSU Digital Commons. For more information, please contactgradetd@lsu.edu. 
A CRITICAL ITEM ANALYSIS OF THE QABF:

DEVELOPMENT OF A SHORT FORM ASSESSMENT INSTRUMENT

\author{
A Dissertation \\ Submitted to the Graduate Faculty of the \\ Louisiana State University and \\ Agricultural and Mechanical College \\ in partial fulfillment of the \\ requirements for the degree of \\ Doctor of Philosophy
}

in

The Department of Psychology

by

Ashvind Nand Singh

B.A., Virginia Commonwealth University, 1999

M.A., Louisiana State University, 2005

May 2008 


\section{ACKNOWLEDGEMENTS}

The encouragement and support of many people contributed to the completion of this dissertation. I am grateful for the assistance and guidance of my dissertation director and advisor, Dr. Johnny L. Matson, Ph.D. I wish to thank my committee members, Dr Wm. Drew Gouvier, Dr. Tracey E. Rizzuto, Dr. Tom E. Davis, III, and Dr. Jianan Wu for their advice and guidance throughout this study. I would like to give special thanks to Dr. Jie Weiss and Dr. Michele Mouttapa for their help and guidance regarding the statistical procedures utilized in this study and to the individuals and staff from Pinecrest Developmental Center in Pinecrest, Louisiana. I am also grateful to Mr. Ryan Thorson who assisted with data collection and the reliability and validity checks.

Finally, I extend my deepest gratitude to my mother and father. Without their

encouragement, sacrifice, support, and patience, I would never have been able to reach this goal. 


\section{TABLE OF CONTENTS}

Acknowledgements .................................................................................................................. ii

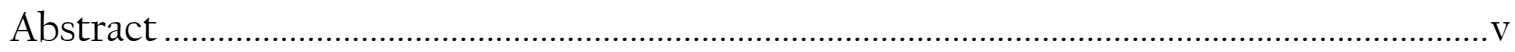

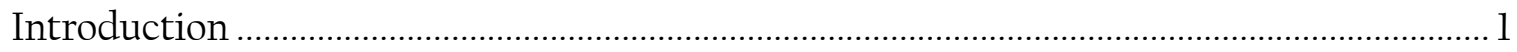

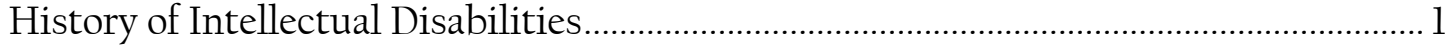

Definition of Intellectual Disabilities ................................................................................... 4

Assessment in Intellectual Disabilities...........................................................................8

Behavioral Correlates of Communication ...................................................................9

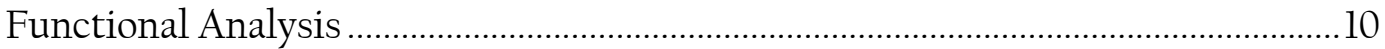

Brief Functional Assessment .........................................................................................11

Difficulties in Assessment ............................................................................................. 13

Scale Development

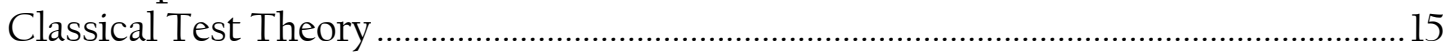

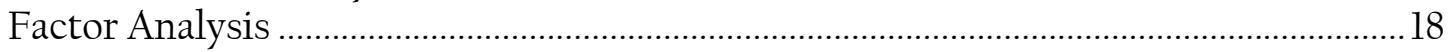

Exploratory Factor Analysis (EFA) ........................................................................ 20

Confirmatory Factor Analysis (CFA) ………………………………………….... 25

Scale Development in Intellectual Disabilities ................................................................. 30

Test Conceptualization …………………………………………………………..... 30

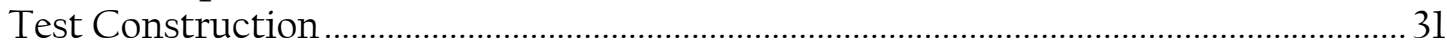

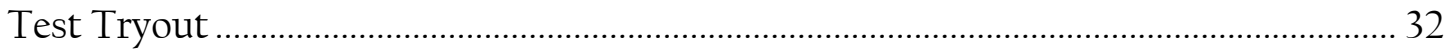

Item Analysis ..................................................................................................................

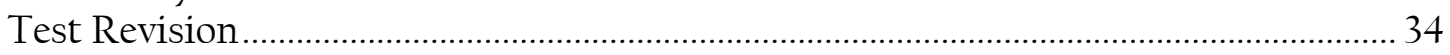

Questions About Behavioral Function (QABF) ……………………………………………... 36

Scope and Development ……………………………………………………………. 36

Psychometric Properties and Utility ……………………………………………………....37

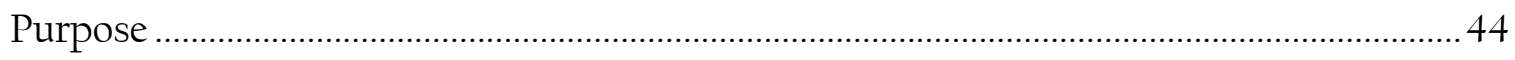

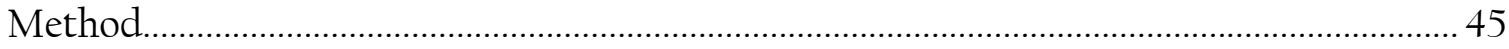

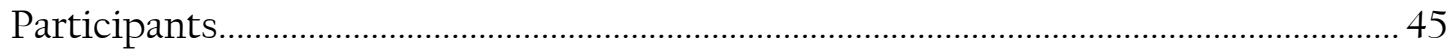

QABF Exploratory Factor Analysis ......................................................................... 45

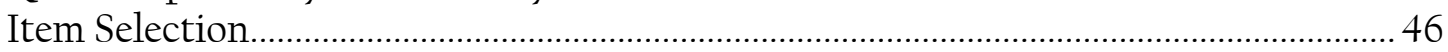

QABF Confirmatory Factor Analysis ............................................................................... 47

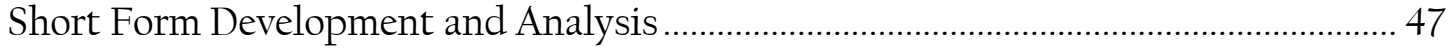

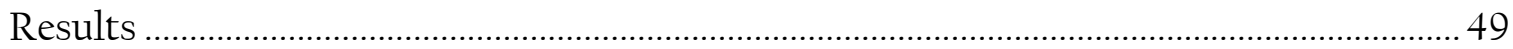

QABF Exploratory Factor Analysis .................................................................................. 49

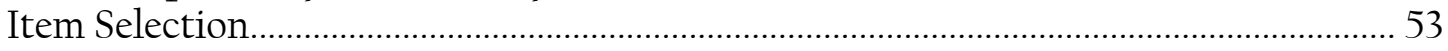

QABF Confirmatory Factor Analysis ...................................................................................56

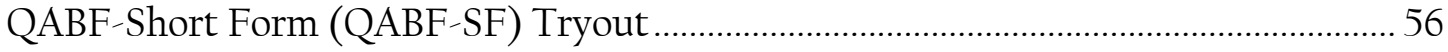




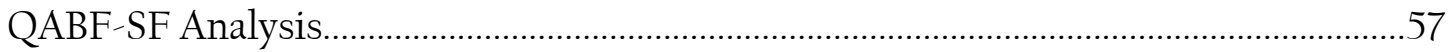

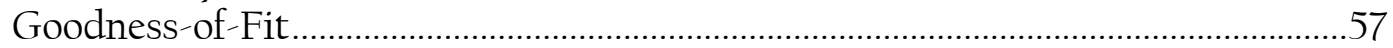

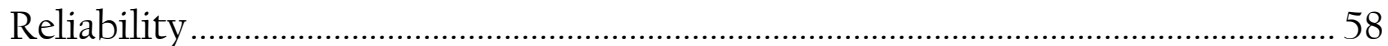

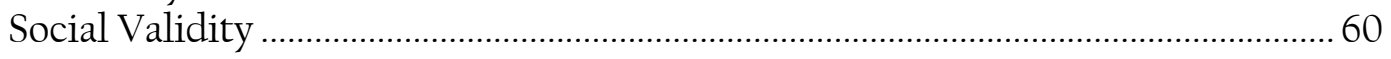

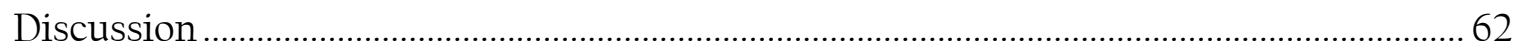

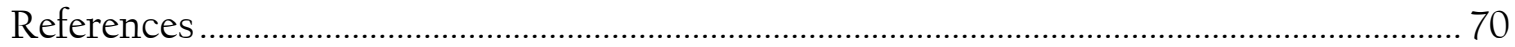

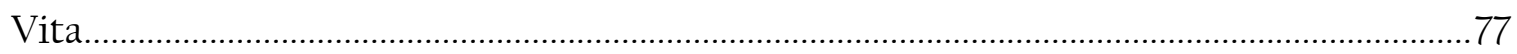




\begin{abstract}
Due to the relative inability of individuals with intellectual disabilities (ID) to provide an accurate and reliable self-report, assessment in this population is more difficult than with individuals in the general population. As such, assessment procedures must be adjusted to compensate for the relative lack of information that the individual can provide and rely more on the behavioral expression of communication. One method commonly used is the indirect functional assessment that utilizes behavior rating scales that have been developed to gather behavioral data in a short time. One of the few empirically derived and psychometrically sound is the QABF, a 25-item questionnaire designed to rate specific behavioral functions and maintaining variables. The purpose of this study was to conduct an item analysis to determine the psychometric properties of the QABF and determine if a short form version could be developed that is both psychometrically valid and reliable, and clinically useful. Results of the item selection procedure indicted that the original 25 items could be reduced by ten. Test tryout procedures showed that the QABF-SF maintained the original five factor structure of the original while maintaining the high degree of reliability and validity. The QABF-SF appears to be a useful tool to aid clinicians in the brief functional assessment of behavior in individuals with ID.
\end{abstract}




\section{INTRODUCTION}

\section{History of Intellectual Disabilities}

One of the first written accounts of ID dates back to around 1500 B.C. in the therapeutic papyri of Thebes, in Egypt (Sheerenberger, 1983). The treatment of individuals with intellectual disabilities (ID) has been typically linked to the current customs and beliefs of the era or culture. For example, infanticide was common practice in ancient Greece and Rome as children suspected of developmental delays were often thrown off cliffs (Biasini, Grupe, Huffman, \& Bray, 1999). The belief was that individuals with moderate to severe physical or mental deficits or handicaps could not hunt, gather, or keep up with the expectations or demands of society. However, those with mild deficits who could positively contribute to their community with minimal assistance were usually allowed to live (Sheerenberger, 1983). By the second century A.D., individuals with disabilities who lived in the Roman Empire were frequently sold and used for entertainment or amusement. This practice began to decline with the dawning of the Christian era, but a movement toward humane treatment for individuals with ID was advocated by all of the early religious leaders such as Jesus, Buddha, Mohammed and Confucius (Sheerenberger, 1983).

The status and treatment of individuals with ID varied greatly during the Middle Ages (476 - 1799 A.D.). Although society had developed more humane practices toward the care of these individuals, there still remained widespread slavery and abandonment (Biasini et al., 1999). It was not until 1690, when John Locke published An Essay Concerning Human Understanding, in which he introduced the idea that individual's were born without innate ideas, that society began to rethink the notion that individuals with ID were untrainable. Locke's work profoundly influenced both the care and training provided to individuals with ID. This was not the only contribution Locke's work made to the treatment of individuals with ID because he 
was also the first to distinguish between ID and mental illness: "Herein seems to lie the difference between idiots and madmen, that madmen put wrong ideas together and reason from them, but idiots make very few or no propositions and reason scarce at all" (Doll, 1962).

The evolution of the care and treatment of individuals with ID took a major step with the works of Jean-Marc-Gaspad Itard and Edouard Seguin (Sheerenberger, 1983). In 1800, Itard was hired by the Director of the National Institutes for Deaf-Mutes in France to work with a young boy named Victor. Apparently, Victor had lived his whole life in the woods of south central France and when captured at the age of 12, was found to be deaf and mute. Itard based his work with Victor on the ideas set forth by Locke and Condillac who emphasized learning through the senses. As such, Itard developed a broad-based educational program for Victor to aid him in developing his senses, intellect, and emotions. After 5 years of training, Victor acquired more skills and knowledge than many of Itard's colleagues believed possible, although he still displayed significant difficulties in language and social interaction. Itard's work and educational approach became widely accepted and spurred a new movement in the education of the deaf. Eventually, the development of this approach led to his work with children with ID. Although Itard did not personally work with these children, he supervised the work of Seguin (Sheerenberger, 1983). Seguin's contribution to the education of children with ID was the development of a comprehensive educational approach known as the Physiological Method that assumed a direct relationship between the senses and cognition.

Seguin's method began with sensory training including vision, hearing, taste, smell, and hand-eye coordination (Biasini et al., 1999). After a series of sensory instructional training modules were completed, the curriculum extended to developing basic self care skills and then onto vocational education that emphasized perception, coordination, imitation, positive reinforcement, memory, and generalization (Biasini et al., 1999). Seguin moved to the United 
States in 1850 and became a major force in the education of individuals with ID. In 1876, he founded an organization known as the American Association on Mental Deficiency, later becoming the American Association on Mental Retardation. Many of the techniques that Seguin pioneered have been modified and are still in use today (Biasini et al., 1999).

Two key developments occurred in the United States over the next 50 years that furthered the education and care movement of individuals with ID: (1) 19 state and 9 privately operated residential training schools were established (Biasini et al., 1999) and, (2) in 1910 Henry Goddard, Director of Research at the Training School in Vineland, New Jersey, translated and published an American version of a newly developed intelligence test by French psychometrist Alfred Binet. The development of the new intelligence test was spurred by the need to determine which children were eligible for and required special education services. The original version of the Binet-Simon Individual Tests of Intelligence was intended to distinguish between subnormal and normal school-aged children and was interpreted in terms of three levels of intellectual disability from most to least disabled: (1) idiocy; (2) imbecility; and (3) moronity (Sheerenberger, 1983).

Another development in the advancement of the treatment and care of individuals with ID was the development of the Vineland Social Maturity Scale in 1935 by Edgar Doll. Specifically, this scale was developed to assess the adaptive and daily living skills of individuals suspected of having ID (Doll, 1953). The emergence of these new techniques to identify and classify individuals with ID gave psychologists and educators hope that it was now not only possible to identify individuals with developmental delays, but also provide them with appropriate care and training in residential schools (Biasini et al., 1999). Due to these new developments, during the early $20^{\text {th }}$ century, residential training schools for individuals with ID proliferated. Unfortunately, some of this proliferation was due to the naive assumption that 
with proper training individuals with ID could be cured. When these training facilities were unable to cure the individuals in their care, they became overcrowded and many students were moved back into society where the focus became special education classes in the community (Biasini et al., 1999). As a result residential and training facilities, both state and private, became custodial living centers (Balthazar \& Stevens, 1975).

The disillusionment with residential treatment centers in the United States and the custodial role they began to serve resulted in the founding of advocacy groups such as the National Association of Retarded Citizens and the President's Commission on Mental Retardation in the 1950's through the 1970's. Also in the 1970's a landmark court case, the Wyatt-Stickney federal court action, helped establish the rights of individuals with ID. Specifically, this class action suit in Alabama established the right to treatment of individuals living in residential care facilities, thus making purely custodial care unacceptable. The Wyatt vs. Stickney case led to several other developments and advances in the care of individuals with ID. Most notable was the creation of the Education of the Handicapped Act passed by Congress in 1975, now titled the Individuals with Disabilities Education Act (IDEA). IDEA guarantees that all children ages 3 to 21 with ID receive appropriate educational services and provides incentives at the state level for the development and provision of service delivery systems.

\section{Definition of Intellectual Disabilities}

The definition of ID has varied greatly over time with early classifications being based on social competence. However, following the development of standardized intelligence tests and adaptive scales in the early 1900's, the definition has become more objective and more focused (Mathias \& Nettlebeck, 1992). In the United States, the elements of the definition of ID were well accepted by 1900 (Sheerenberger, 1983). These elements included onset in childhood, significant intellectual or cognitive limitations, and an inability to adapt to the demands of 
everyday life. In 1910, the American Association on Mental Deficiency proposed a definition that referred to individuals with ID as feeble-minded, meaning that their development had halted at an early age or in some way was inadequate thus making it difficult for these individuals to keep pace with their peers and manage their own lives independently (Committee on Classification, 1910). The Committee defined three levels of impairment: (1) idiot, individuals whose development is arrested at the level of a 2 year old; (2) imbecile, individuals whose development is equivalent to that of a 2 to 7 year old at maturity; and (3) moron, individuals whose mental development is equivalent to that of a 7 to 12 year old at maturity.

According to Yepsen (1941), over the next 30 years the definition of ID focused on three aspects of development: (1) the inability to learn to perform common acts, (2) deficits or delays in social development/competence, and (3) low IQ. However, as the services provided to individuals with ID grew and were increasingly being funded by public money, there was an increased need for more accurate and clearer set of standards to identify individuals who were eligible for public services. Thus, it became apparent that new levels needed to be defined and linked to IQ scores on the newly revised Stanford-Binet intelligence test (Scheerenberger, 1983). Thus, in 1959, a new classification system of ID was developed by the American Association on Mental Deficiency. The new definition stated that an individual must demonstrate impaired adaptive functioning that originated before the age of 16 and consisted of five levels of ID linked to IQ scores: (1) borderline (IQ of 83-67); (2) mild (IQ of 66-50); (3) moderate (IQ of 49-33); (4) severe (IQ of 32-16); and (5) profound (IQ of 16 or below). Although this definition covered three areas, only age of onset and IQ were measurable with existing psychometric techniques as deficits in adaptive functioning were typically based on subjective interpretations although the Vineland Social Maturity Scale was available (Scheerenberger, 1983).

In 1973, the definition of ID was revised due to concerns over misidentification, particularly in minority populations (Grossman, 1973). In the new definition, the classification 
of borderline IQ was eliminated. Further, the upper IQ boundary was changed from $<85$ to $\leq 70$ which significantly reduced the number of individuals who were previously identified as mentally retarded. In 1977, the definition was again revised and the upper IQ limit was set at 7075 to account for measurement error (Grossman, 1977). Thus, IQ scores of 71 through 75 were only consistent with ID when significant deficits in adaptive functioning were present. The new definition contributed a new feature to the understanding of ID, that is, impairment in adaptive functioning (Scheerenberger, 1983). Thus, researchers began focusing on the elements of adaptive functioning: basic motor and self-help skills; learning; and social adjustment. New methods for measuring adaptive functioning were developed and two rating scales were introduced: the AAMD Adaptive Behavior Scale (ABS; Nihira, Foster, Shallhaas, \& Leland, 1969) and the Vineland Adaptive Behavior Scale (VABS; Sparrow, Balla, \& Cicchetti, 1984).

Currently, in the Diagnostic and Statistical Manual-IV-Text Revision (APA, 2000) developmental disability is characterized by three criteria: (l) sub-average intellectual functioning, (2) significant limitations in adaptive skills, and (3) onset before age 18. Intellectual functioning is defined by the intelligence quotient (IQ) and is obtained with the use of one or more standardized administered tests (i.e., Stanford-Binet, Wechsler Intelligence Scales). Sub-average intellectual functioning is defined as an IQ score of 70 or below, or two standard deviations below the mean. However, although IQ is a defining factor of intellectual functioning, there is widespread dissatisfaction with the reliance on measures of intelligence because of co-variation with socioeconomic factors (Flanagan, Genshaft, \& Harrison, 1997). These concerns added to those of Blatt and Kaplan (1966) who elucidated the problems on relying too heavily on IQ, thus highlighting the need for the consideration of adaptive behavior.

A limitation in adaptive functioning constitute the second criteria associated with the diagnosis of ID and refers to an inability to cope with life's demands typical of someone of the 
same age, background, and community surroundings (APA, 2000). This criterion requires significant skill deficits in two or more of the following areas: communication, self-care, home living, social/interpersonal skills, use of community resources, self-direction, functional academic skills, work, leisure, health, or safety (APA, 2000). Scales such as the Vineland Adaptive Behavior Scales (VABS) have been designed to assess levels of adaptive functioning (Sparrow, Balla, \& Cicchetti, 1984).

The third criterion required for the diagnosis of developmental disability is onset before the age of 18. Specifically, the DSM-IV-TR (APA, 2000) states that the age of onset is typically linked to the etiology and level of intellectual impairment; that is, more severe ID tend to be recognized earlier than milder disabilities. Further, disabilities associated with syndromes such as Fragile $\mathrm{X}$ are usually diagnosed at birth whereas disabilities with an unknown etiology tend to be diagnosed later in life (Greenspan, 1999).

For the most part, the different levels of ID are still associated with ranges in IQ scores. The DSM-IV-TR (APA, 2000) describes four levels of ID: (1) mild (IQ 50-55 to 70); (2) moderate (IQ 35-40 to 50-55); (3) severe (IQ 20-25 to 35-40); and (4) profound (IQ below 20-25). Mild ID accounts for approximately 85\% of all individuals with ID (APA, 2000; Greenspan, 1999). Individuals in this range typically develop social and communication skills, have minimal sensorimotor impairment, and are often indistinguishable from non-disabled individuals. Although individuals with mild ID usually learn and maintain social and vocational skills, they may require supervision, guidance, and assistance.

Individuals in the moderate range account for approximately $10 \%$ of all individuals with ID (Greenspan, 1999). These individuals typically acquire communication skills during childhood and with moderate supervision profit from vocational training and can attend to their own personal needs. The DSM-IV-TR (APA, 2000) states that individuals with moderate ID can, with moderate supervision, generally adapt well to life in the community. 
The third level of impairment, severe, accounts for approximately $3 \%$ to $4 \%$ of all individuals with ID (Greenspan, 1999). Individuals in this range typically do not develop communication skills during early childhood, but may learn to talk and gain some simple selfcare skills during school-age. These individuals require supervision in most settings (APA, 2000).

The fourth, and most severe, level of ID is the profound range. Individuals with profound disability account for approximately only $1 \%$ of all individuals with ID and their disability often stems from an identified neurological condition (Greenspan, 1999). Individuals in this range often have considerable impairment in sensorimotor functioning, communication, and self-care skills; although these areas may be improved with appropriate training. However, these individuals generally require highly supervised and individualized care (APA, 2000).

\section{Assessment in Intellectual Disabilities}

Many individuals with intellectual disabilities (ID) are non-verbal and must use other means to communicate or control their environment. Researchers suggest that individuals with ID who have limited communication skills rely primarily on expressive behavior to communicate their wants and needs (Dura, 1997; Durand \& Carr, 1991). In fact, these limited skills can lead to communication in the form of socially inappropriate or maladaptive behavior such as aggression, self-injury, or self-stimulatory behavior (Menolascino, Levitas, \& Greiner, 1986). Therefore, aggression, property destruction, self-injury and other socially inappropriate behaviors may serve a functional purpose for an individual (Carr \& Durand, 1985). However, in order to understand why a maladaptive behavior is occurring, and therefore its functional purpose, one must identify the contingencies that maintain it (Bandura, 1969; Skinner, 1953). In order to identify maintaining contingencies of behavior, psychologists typically assess the antecedents and consequences that are functionally related to it. 
$\underline{\text { Behavioral Correlates of Communication }}$

Researchers have identified five primary maintaining functions of maladaptive behavior; these are attention, escape, tangible reinforcement, physical discomfort, and non-social reinforcement (Carr, 1994; Derby et al., 1992; Lowry \& Sovner, 1991; Taylor, Ekadahl, Romancyzk, \& Miller, 1994). For example, an individual may engage in physical aggression in the form of hitting when he or she wants somebody to interact with them, either in a positive or negative manner. In this case, the function of the behavior for the individual is to get attention. In another situation, the same individual may hit to get out of being somewhere or doing something that they do not like. This individual has learned that if they hit someone, staff will typically remove them from the environment or stop making the demand. In this situation, the individual's hitting is maintained by being allowed to escape a situation or task they do not like. In another example, when an individual engages in maladaptive behavior and is rewarded by getting something he or she wants such as a favorite toy, the maintaining consequence of the behavior is gaining access to the toy. The function of the individual's behavior is to gain access to a tangible. Another communicative function of maladaptive behavior is conveying physical discomfort; this is particularly salient in individuals with ID who often have multiple comorbid health concerns. For example, sometimes an individual will hit one of their ears repeatedly because he or she has an earache or are trying to tell you that they are physically sick. Lastly, an individual may engage in maladaptive behavior for non-social reasons, such as to attenuate pain, avoid boredom, or provide themselves with some type of stimulation. Currently, there are two primary assessment methods used to gather information and data regarding the functional behavior of individuals with ID: 1) functional analysis, and 2) brief functional assessment. Functional Analysis

Haynes and O'Brien (1990) proposed that a functional analysis should be defined as "the identification of important, controllable, causal functional relationships applicable to a specified 
set of target behaviors for an individual." Generally, the functional analysis procedure involves systematically manipulating the antecedents and consequences of behavior and inferring the function from the resulting rate change (Iwata, Dorsey, Slifer, Bauman, \& Richman, 1982). Thus, functional analysis procedures can be seen as a process to aide clinicians to systematically identify predictive relationships between events in the environment and the occurrence of target behaviors such as physical or verbal aggression, pica, self-injury, or property destruction.

The development and introduction of functional analysis is typically attributed to Iwata et al. (1982) and has been widely used in the research literature to study maladaptive behaviors in individuals with ID. The procedure for the functional analysis of behavior is fairly simple and straightforward. It begins with identification of the target behavior. After the target behavior has been identified, it is operationalized and baseline data are collected and analyzed. The next step is to conduct an experimental functional analysis. After the experimental analog sessions are conducted the data are compared with baseline data. The clinician then develops and implements a behavioral treatment that targets an individual's maladaptive behavior and uses skills training to teach the individual functionally alternative adaptive behavior. As such, the prescribed interventions are typically are based on the consequences of the behavior and do not the individuals motivation.

The defining feature of functional analysis is the systematic identification of the environmental determinates of behavior (Iwata, 1994). In effect, the functional analysis procedure isolates and controls those contingencies the psychologist feels may maintain an individual's behavior using standardized procedures (Iwata et al., 1982; Wacker et al., 1990). The purported advantage of using this type of methodology is that the clinician can use the results to select the most effective treatment; that is, at least theoretically, based on the function of an individual's behavior and the conditions that maintain it. Basing treatment on the function 
of the behavior is important because in order for treatment to be effective, the desired replacement behavior must serve the same function as the target behavior (Wacker et al., 1990).

When developing a behavioral treatment plan, a problem occurs when the function of the replacement behavior does not match the function of the original target behavior. In fact, researchers have suggested that treatments based on a general assessment strategy, such as functional analysis, is likely to fail if the antecedent and consequent factors that are chosen do not adequately match those in the natural environment (Sturmey, 1995). Further limiting functional analysis is fact that a maladaptive target behavior may serve several functions (Gable, 1996) or be maintained by more than one mechanism such as positive and negative reinforcement (Durand \& Carr, 1992; Smith, Iwata, Vollmer, \& Zarcone, 1993). These factors add to the time and cost of implementing the functional analysis procedure and tend to produce confusing or unclear results. Taking into account these considerations, as well as some of the ethical implications of eliciting potentially dangerous behavior, it is no wonder that clinicians are moving past the functional analysis and exploring assessment options that are more cost effective, and less time and resource consuming, such as the brief functional assessment.

\section{$\underline{\text { Brief Functional Assessment }}$}

Several steps are involved in conducting a brief functional assessment. The first step is developing an operational definition of the target behavior in observable and measurable terms. Second, an indirect functional assessment is begun with a structured interview conducted with those staff and other individuals who spend a significant amount of time with the client (see O’Neill, Horner, Albin, Storey, \& Sprague, 1990). Individuals who can be interviewed include, but are not limited to, direct care staff, teachers, parents, and friends. Third, rating scales are used to help determine environmental variables maintaining an individual's behavior. The goal of interviews and informal data collection is to develop a hypothesis regarding what function the behavior serves for the individual and factors that may maintain it. 
Fourth, after the indirect assessment has been performed and the data analyzed, the clinician should have developed several hypotheses as to the function of the target behavior. However, as the informal assessment utilized indirect assessments, no direct observations of the target behavior have been conducted. As such, a direct assessment is then usually undertaken; unless the target behavior is of low frequency and direct observation is not feasible. The goal of the direct assessment is to clarify environmental variables that may be maintaining the individuals target behavior and provide other clinically relevant information. This type of assessment involves analyzing the frequency of the behavior over specific time intervals, time periods, patterns, and contexts.

Finally, the psychologist looks for correlations among information gathered from the interviews, data from the rating scales, and direct observations. Often, the combination of the data will yield possible functions of the target behavior. Based on this information, a behavioral intervention is designed to target the maladaptive behavior that alters antecedent events that elicit the behavior (Weeks \& Gaylord-Ross, 1981), reinforces appropriate alternative behavior (Carr \& Durand, 1985), or eliminates access to reinforcement via extinction (Iwata, Pace, Kalsher, Cowdery, \& Cataldo, 1990).

Several methods for collecting data during a brief functional assessment have been identified in the literature. One of the most prominent is called scaling and utilizes behavior rating scales. Behavior rating scales are a type of rating scale that collects data via a third-party informant familiar with the individual being assessed to respond to questions about the individual's behavior and its possible functions. Questions on the behavior rating scale typically address observable behavior, rely less on subjective impressions, and are designed to assess various constructs, skills, deficits, and other observable behaviors. Examples of behavior rating scales commonly used are the Diagnostic Assessment of the Severely Handicapped-II (DASH-II; 
Matson, 1995a), the Behavior Problems Inventory (BPI; Rojahn, Polster, Mulick, \& Wisniewski, 1989), the Aberrant Behavior Checklist (ABC; Aman \& Singh, 1986), and the Assessment of Dual Diagnosis (ADD; Matson \& Bamburg, 1998).

Adaptive functioning scales also utilize indirect assessment methods to gather data during a brief functional assessment. Some common adaptive functioning rating scales are the Vineland Adaptive Behavior Scale (VABS; Sparrow, Balla, \& Cicchetti, 1984) and the Adaptive Behavior Scale (ABS; Nihira, Leland, \& Lambert, 1993). Assessment of social skills excesses and deficits can also be accomplished using an indirect assessment. One widely used measure is the Matson Evaluation of Social Skills in Individuals with sEvere Retardation (MESSIER; Matson, 1995b). Rating scales that identify functional variables that maintain problem behavior also routinely utilize indirect assessment methods and are often used instead of a formal functional analysis. Two behavior rating scales that are commonly used in a brief functional assessment include the Motivational Assessment Scale (MAS; Durand \& Crimmins, 1988), and the empirically validated Questions About Behavioral Function (QABF; Vollmer \& Matson, 1995).

\section{Difficulties in Assessment}

Due to the relative inability of individuals with ID to provide an accurate and reliable self-report, assessment in this population is more difficult than with individuals in the general population. As such, assessment procedures must be adjusted to compensate for the relative lack of information that the individual can provide. In the assessment of individuals with ID, examiners are forced to rely more heavily on observable behaviors exhibited by the individual and/or reports of observable behavior. Complicating the assessment of behavior problems among individuals with ID is the lack of requisite time, training, personnel, or resources practitioners have to conduct in vivo functional analyses. Thus, the brief functional assessment has emerged as the assessment method of choice for professionals who work in large hospitals and not in small research laboratories. 
Although less resource consuming than functional analysis, the process of conducting a brief functional assessment can still be an exhaustive process, depending on the nature and number of the individual's behavior problems and the quality of the reinforcement maintaining them. Thus, even within the brief functional assessment, measures need to be as short as possible while providing enough data to be clinically useful. For example an individual may engage in the maladaptive behavior across contexts, or the behavior may serve multiple functions for the individual depending on the environment. Another concern is the quality of the data gathered. In order to obtain the most reliable and valid information in less than ideal circumstances, items chosen for inclusion on any measure must be the best ones to access the desired information. Added to these constraints are issues of staff training (i.e., clinical psychologist versus front line staff), patterns of staffing support (i.e., high turnover, staff coverage, and mandatory overtime) and systems issues related to the provision of care. 


\section{SCALE DEVELOPMENT}

The assessment of individual differences has a very long history dating back to the use of formal testing procedures for selection and performance appraisal by the Chinese civil service in 2200 B.C. (Bowman, 1989). The system developed by the Chinese eventually became the model

followed by British, French, and German governments of the $19^{\text {th }}$ century. Also, during this time, the science of testing and measurement gained significant momentum with the rise in interest in individual differences. Several factors spurred interest in this new science, including the work of the English naturalist Charles Darwin, the experimental psychological works of Wilhem Wundt and Hermann Ebbinghaus, the study of intelligence in France by Alfred Binet and Theodore Simon, and the work of English biologist Sir Francis Galton (Kline, 2005). One of the seminal events in testing and test development occurred when Binet and Simon were commissioned by the French minister of public education to develop a test to identify those children who would not or could not benefit from traditional instruction in the regular school system (Kline, 2005). Their work resulted in the first formal intelligence test and the development of formal test theory.

\section{Classical Test Theory}

In order to provide an overview of classical test theory, it is first important to introduce five measurement problems that Crocker and Algina (1986) posit as common to all psychological assessments and that all test developers must cope with: (1) no single approach to the measurement of any construct in universally accepted; (2) psychological measurements are usually based on limited samples of behavior; (3) the measurement is always subject to error; (4) the lack of well-defined units on the measurement scales poses still another problem; and (5) psychological constructs cannot be defined only in terms of operational definitions but must also have demonstrated relationships to other constructs or observable phenomena. 
Classical test theory is a body of related psychometric theories that predict outcomes of psychological testing, such as the difficulty of items or the ability of test-takers (Cohen \& Swerdlik, 1998). Generally, the aim of classical test theory is to understand and improve the reliability of psychological tests. The term classical refers not only to the chronology of these models but also contrasts with the more recent psychometric theories, generally referred to collectively as Modern test theory, also referred to as Item Response theory (Kline, 2005).

While there are several types of classical test theories, their common foundation rests on the assumption that an individual's observed raw scores $(X)$ are composed of true $(T)$ and error (E) scores. This definition is formally stated as:

$$
\chi=\mathrm{T}+\mathrm{E}
$$

However, classical test theory is never used to analyze individual test scores; rather, the focus of the theory is on properties of test scores relative to populations of people (Kline, 2005). Classical test theory is concerned with the relations between the three variables $\mathcal{X}, \mathrm{T}$, and $\mathrm{E}$ in the population. These relationships are used to say something about the quality of test scores and, thus, of the test itself (Crocker \& Algina, 1986). In this regard, the most important concept within test theory is that of reliability. The reliability of the observed test score $\mathcal{X}$, which is denoted as $\rho_{X T}^{2}$, is defined as the ratio of true score variance $\sigma_{T}^{2}$ to the observed score variance $\sigma_{X}^{2}$

$$
\rho_{X T}^{2}=\frac{\sigma_{T}^{2}}{\sigma_{X}^{2}} .
$$

Because the variance of the observed scores can be shown to equal the sum of the variance of true scores and the variance of error scores, this is equivalent to: 


$$
\rho_{X T}^{2}=\frac{\sigma_{T}^{2}}{\sigma_{X}^{2}}=\frac{\sigma_{T}^{2}}{\sigma_{T}^{2}+\sigma_{E}^{2}} .
$$

This equation, which formulates a signal-to-noise ratio, has intuitive appeal for statisticians and test developers (Kline, 2005). The appeal is that as the reliability of test scores becomes higher, the proportion of error variance in the test scores becomes lower and vice versa. The reliability is equal to the proportion of the variance in the test scores that we could explain if we knew the true scores. The square root of the reliability is the correlation between true and observed scores.

However, reliability is not, as is often suggested in textbooks, a fixed property of tests, but a property of test scores relative to a particular population (Kline, 2005). This is because test scores will not be equally reliable in every population. For example, the reliability of test scores will be lowered by restriction of range. Thus, IQ-test scores that are highly reliable in the general population will be less reliable in a population of college students. Further, test scores are perfectly unreliable for any given individual because the true score is a constant at the level of the individual, which implies it has zero variance, so that the ratio of true score variance to observed score variance, and hence reliability, is zero. The reason for this is that, in the Classical test theory model, all observed variability in an individual's scores is random error by definition. Thus, Classical test theory is relevant only at the level of populations and not at the level of individuals (Crocker \& Algina, 1986).

Reliability cannot be estimated directly because that would require one to observe the true scores, which according to Classical test theory is impossible (Cohen \& Swerdlik, 1998; Kline, 2005). However, estimates of reliability can be obtained by other means. One way of doing this is by constructing a parallel test or using parallel test forms. A parallel test is a test that, for every individual, yields the same true score and the same observed score variance as the 
original test (Crocker \& Algina, 1986; Lord, 1959). The estimation of reliability by the use of parallel tests is cumbersome because parallel tests are very hard to come by and the method is rarely used. Instead, researchers use a measure of internal consistency known as Cronbach's alpha (Cohen \& Swerdlik, 1998; Crocker \& Algina, 1986; Kline, 2005). Cronbach's alpha can be shown to provide a lower bound for reliability under rather mild assumptions (Cronbach, 1951). Thus, the reliability of test scores in a population is always higher than the value of Cronbach's alpha in that population. Researchers have found that this method of estimating reliability to be more empirically feasible and, as a result, it is very popular. As noted previously, the exercise of Classical test theory is performed to arrive at a suitable definition of reliability. Reliability is supposed to say something about the general quality of the test scores in question (Crocker \& Algina, 1986). The general idea is that, the higher the reliability, the better the test. Although Classical test theory does not say how high reliability is supposed to be, generally a value over .80 is deemed acceptable while a value over .90 is good (Kline, 2005). Values between .70 and .80 are seen as mediocre but still acceptable and values below .70 are considered bad (Kline, 2005).

By far, Classical test theory is the most influential theory of test scores in the social sciences (Crocker \& Algina, 1986; Kline, 2005). However, in the field of psychometrics, the theory has been superseded by the more sophisticated models in Item Response theory. However, Item Response theory models have been very slow to catch on in mainstream research. One of the main reasons for the slow acceptance and lack of wide use of Item Response theory is the lack of availability of user-friendly software. For example, Item Response theory is not included in standard statistical packages, such as SPSS.

\section{Factor Analysis}

A common procedure used in the development of tests that measure individual differences is the factor analysis. This procedure has its origins in the study of human 
intelligence and was devised as a method for comparing the outcomes of objective tests and to construct matrices to define correlations between these outcomes and finding the factors that are responsible for these results (Thompson, 2004). Historically, the development of factor analysis within the field of psychology is typically credited to Charles Spearman who discovered that school children's scores on a wide variety of seemingly unrelated subjects were positively correlated. This finding led him to postulate that a general mental ability, or $g$, underlies and shapes human cognitive performance. His postulate now enjoys broad support in the field of intelligence research, where it is known as the $g$ theory (Thompson, 2004).

In the late 1940's, Raymond Cattell expanded on Spearman's idea of a two-factor theory of intelligence after performing his own tests and factor analysis. He used a multi-factor theory to explain intelligence and address alternate factors in intellectual development, including motivation and psychology (Cattell, 1950). Cattell also developed several mathematical methods for adjusting psychometric graphs, such as his scree test and similarity coefficients and lead to the development of his theory of fluid and crystallized intelligence. Cattell was a strong advocate of factor analysis and believed that all theory should be derived from research and the use of empirical observation and objective testing to study human intelligence (Lohman, 1989). Since the pioneering work of Spearman and Cattell, researchers have continued to use the factor analysis procedure to develop and refine both theories regarding individual differences and the tests that are used to measure them.

The basic hypothesis of the factor analysis procedure is that within any given domain of human performance there exist a small number of common factors that influence the numerous surface attributes of an individual, that is, attributes that can be observed and measured (Kim \& Mueller, 1978a). For example, in the domain of mental abilities, tests could be developed that measure different kinds of attributes such as addition problems, spelling, or memory; each one of 
these test represent a surface attribute. A cornerstone of this basic hypothesis with regard to factor analysis and surface attributes is that there exist internal attributes, the unobservable characteristics of individuals that differ between individuals in degree and are more fundamental that surface attributes (Kim \& Mueller, 1978a). A set of surface attributes measured by a given test is referred to as a battery of surface attributes. Within the surface attribute of mental ability, internal attributes could be numerical, verbal, or performance ability.

While internal attributes cannot be directly measured, they are reflected when one obtains a measure of the surface attribute. These internal attributes are often referred to as factors. Within internal attributes, or factors, there are two types: common and specific. Common factors are those internal attributes that affects more than one surface attribute in the selected battery. For example, if the selected battery of surface attributes within a test includes more than one that is influenced by verbal ability (e.g. both a spelling and reading test) then verbal ability is a common factor. Specific factors, on the other hand, only influence one of the surface attributes within the battery. While there may be a number of specific factors for any given surface attribute, their influences can be viewed as being combined into a single specific factor. The essential principle is that internal factors affect surface attributes in a systematic manner (Gorsuch, 1990). Thus, the mathematical procedures of the factor analysis are utilized to identify and clarify the nature of this relationship. Typically during scale development researchers will use two types of factor analysis: exploratory and confirmatory.

\section{Exploratory Factor Analysis (EFA)}

Generally, the EFA is used to discover the factor structure of a measure and examine its internal reliability. EFA is often recommended when researchers have no hypotheses about the nature of the underlying factor structure of their measure. The EFA has three basic decision points: (1) choosing an extraction method, (2) choosing a rotation method, and (3) deciding the 
number of factors. In a recent PsychINFO search, over 1700 studies were found that used EFA procedures over a two year period (Costello \& Osborne (2005). However, while the EFA presents the norm in literature, it is a complex procedure with few absolute guidelines and a variety of options (Costello \& Osborne, 2005). Adding to this confusion is that EFA options vary across software packages, and in most cases, these options are not very well defined. Further, study design, data properties, and the question of interest all have a bearing on which procedure will yield the maximum benefit (Costello $\&$ Osborne, 2005). Literature suggests that there are four primary issues that should be considered when determining the best EFA procedure: 1) method of extraction, 2) number of factors to retain for rotation, 3) orthogonal vs. oblique rotation, and 4) adequate sample size.

The default method of extraction of many popular statistical packages, including SPSS and SAS, is the Principle Components Analysis (PCA). However, statisticians disagree on the utility of PCA, as it is not a true method of factor analysis due to the partition of shared variance, and when it should be used (Bentler \& Kao, 1990). Some argue that the use of PCA should be restricted in favor of a true factor analysis method (Snook \& Gorsuch, 1989). However, others argue that there is almost no difference between PCA and factor analysis, or that PCA is the preferable method of analysis (Velicer \& Jackson, 1990). The choice of extraction method is often left up to the researcher, although given that PCA is the default method of extraction in statistical packages, PCA is the most commonly used method of extraction seen in the literature (Costello \& Osborne, 2005).

Besides PCA, there are several other factor analysis extraction methods available. For example, SPSS has five in addition to PCA: unweighted least squares, generalized least squares, maximum likelihood, principal axis factoring, alpha factoring, and image factoring. However, information regarding the relative weakness and strengths of each of these extraction methods is 
scarce (Costello \& Osborne, 2005). Complicating matters further, there are no exact names for several of these methods; it is often hard to figure out which method is being described in many textbook or journal articles; and the availability of specific methods within software packages is sometimes hard to determine. Recently, Fabrigar, Wegener, MacCallum and Strahan (1999) argued that if the data being analyzed are relatively normally distributed, the Maximum Likelihood (ML) extraction method is the best choice because "it allows for the computation of a wide range of indices of the goodness-of-fit of the model [and] permits statistical significance testing of factor loadings and correlations among factors and the computation of confidence intervals." (p. 277). If the assumption of normality of the data is violated, these authors recommend one of the principle factor methods; in SPSS a procedure called "Principle Axis Factors (PAF)" (Fabrigar et al., 1999). In general, literature suggests that ML or PAF will give the best results, depending on whether the data are generally normally distributed or not (Costello \& Osborne, 2005).

The second issue concerns the number of factors to retain. Both underextraction and overextraction of the factors to be retained can have deleterious effects on the results (Costello $\&$ Osborne, 2005). The most common approach to deciding the number of factors in common statistical packages is to retain all factors with eigenvalues greater than 1.0 and to then generate a scree plot, a two dimensional graph with factors on the $\mathrm{x}$-axis and eigenvalues on the $\mathrm{y}$-axis. Eigenvalues represent the variance accounted for by each underlying factor. They are not represented by percentages but scores that total to the number of items. For example, a 12 -item scale will theoretically have 12 possible underlying factors; each factor will have an eigenvalue that indicates the amount of variation in the items accounted for by each factor. Eigenvalues are typically arranged in a scree plot in descending order: 


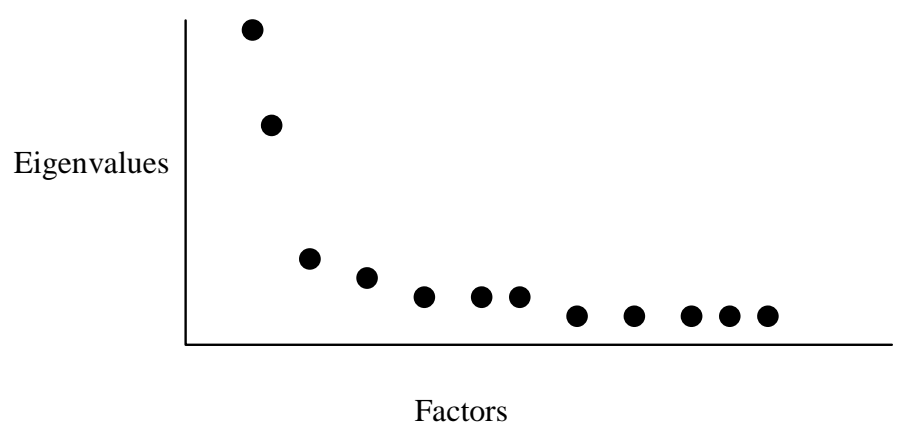

From the scree plot above, it is evident that the first two factors account for most of the variance and have the highest eigenvalues, thus the remaining factors all have small eigenvalues. However, there is broad consensus among psychometric researchers that this is among the least accurate methods for selecting the number of factors to retain (Velicer \& Jackson, 1990). There are alternative tests for factor retention available, including the scree test, Velicer's MAP criteria, and parallel analysis, however, they are not available in the most frequently used statistical software packages and must be calculated by hand (Costello $\&$ Osborne, 2005). Thus, researchers rely on the default method found in most software packages; that is, eliminating those factors with eigenvalues less than 1.0.

Once an initial solution is obtained and the number of factors for retention has been determined, the next decision is the method used for rotation. The goal of factor rotation is to maximize high loadings and minimize low loadings so that the simplest possible structure is achieved; in other words, to mathematically simplify and clarify the data structure (Child, 1990). Thus, rotation serves to make the output more understandable and is usually necessary to facilitate the interpretation of factors. The sum of eigenvalues is not affected by rotation, but rotation will alter the eigenvalues and percent of variance explained by particular factors and will change the factor loadings. Since alternative rotations may explain the same variance but 
have different factor loadings, and since factor loadings are used to intuit the meaning of factors, different meanings may be ascribed to the factors depending on the rotation, a problem often cited as a drawback to factor analysis (Child, 1990). As with extraction methods, there are a variety of choices. However, there are two basic types of rotation: orthogonal and oblique. In orthogonal rotation, the factors are assumed to be uncorrelated with one another; this is the default setting in most statistical packages. These orthogonal methods produce factors that are uncorrelated and include: varimax, quartimen, and promax rotations. Oblique rotation derives factor loadings based on the assumption that the factors are correlated.

According to conventional wisdom, researchers in the social sciences are advised to use orthogonal rotation because it produces more easily interpretable results (Costello \& Osborne, 2005). Ideally, after the researcher has rotated the factor loadings a clearer picture of the relationship between the internal factors and the surface attribute should emerge (Kim \& Mueller, 1978b). The researcher then knows the factor structure of their test, or construct, and can then label the factors that contribute the most variance to an individual's performance on the surface attribute being measured. Orthogonal rotation output is only slightly simpler than that of an oblique rotation. In SPSS output, the rotated factor matrix is interpreted after orthogonal rotation. However, the substantive interpretations of orthogonal and oblique rotations are essentially the same (Costello \& Osborne, 2005).

The last issue within the choice of EFA procedure appropriateness concerns sample size. After recently reviewing articles listed in PsychINFO that reported using some form of principle components or exploratory factor analysis over the past two years, Costello and Osborne (2005) suggest that the best determinant of subject size is the ratio of subjects to items. These authors also found that strict rules regarding sample size for EFA have largely disappeared. Other researchers have suggested that adequate sample size is partially determined by the nature and 
availability of the data (Fabrigar et al, 1999). Generally, researchers agree that the stronger the data, the smaller the sample can be for accurate analysis (Mulaik, 1990) and that a factor with three or fewer items is generally weak and unstable (Costello \& Osborne, 2005).

Confirmatory Factor Analysis (CFA)

Both the EFA and CFA are statistical procedures used to examine the internal reliability of a measure; both are used to investigate the theoretical constructs, or factors, that might be represented by a set of items; either can assume the factors are uncorrelated; and both are used to assess the quality of individual items. However, there is one major difference between exploratory and confirmatory factor analysis. The EFA finds the one underlying factor model that best fits the data, whereas the CFA allows a researcher to impose a predetermined factor model on the data and see how well the model explains responses to the measure. With the EFA, the researcher lets the observed data determine the underlying factor a posteriori, that is reasoning inductively to infer a model from observed data. With the CFA, the researcher derives a factor model a priori, that is reasoning deductively to hypothesize a structure beforehand. Thus, the EFA represents a tool for theory building, while the CFA represents a tool for theory testing (Bollen, 1989).

The CFA builds upon Classic Test Theory. As with EFA, in CFA each response in a data set is considered to be an observed indicator of one or more underlying latent constructs, or factors. The CFA model assumes that there are two main sources of variation in the responses to the measure of interest. Specifically, individuals' scores on measured variables are assumed to be influenced by latent underlying factors and by unique-measurement error, or the influence of unmeasured variables and random error (Bryant $\&$ Yarnold, 1995). Further, whereas the EFA assumes that the unique errors in the observed indicators are uncorrelated with one another, the CFA allows these measurement errors to be either independent or correlated. Thus, with CFA 
one can parcel out the error variance that variables share as a result of common methods of assessment to examine relationships between variables independent of both unique and correlated measurement error (Bryant \& Yarnold, 1995).

As with the EFA procedure, the strength of the relationships between variables in the CFA are affected by sample size, as are estimates of measurement error variance and the contribution of random error. These relationships are a result of mathematically calculating an estimate of one statistic from an estimate of another (Good, 1973), and the limitations or restrictions inherent to this procedure. Limitations such as these are known as degrees of freedom and are directly related to sample size, $n$, where $n$ can be considered one individual piece of information and the sum of these pieces of information can be used to estimate either model parameters or variability (Toothaker \& Miller, 1986). However, when statistics such as model parameters or variability are estimated, one loses precision every time a statistic is calculated (Jaccard \& Becker, 1990). Degrees of freedom are a measure of the amount of information from the sample data that has been used to calculate the particular statistic (Jaccard \& Becker, 1990). Statisticians mathematically define degrees of freedom as the number of observations minus the number of necessary parameters, or $n-1$. For example, if there are four numbers ( $a, b, c$, and d) that must add up to a total of $\mathrm{m}$; and you are free to choose the first three numbers at random but the fourth must be chosen so that it makes the total equal to $\mathrm{m}$, the degrees of freedom is three. Generally, degrees of freedom are less influential as sample size increases and the distribution of the sample approaches normal. Specifically, literature suggests that when the sample size of the test statistic is less than 30 , the distribution of that test statistic cannot be guaranteed to be normal (Galfo, 1985).

As previously noted, the technical and procedural aspects of the EFA and CFA are similar. However, with the EFA researchers decide on the number of factors by examining 
output from a principal components analysis. With the CFA, researchers must specify the number of factors a priori, the principle difference between the EFA and the CFA (Kim \& Mueller, 1987b). That is, the CFA requires that a particular factor structure be specified in advance and the researcher indicates which items load on which factor while the EFA allows all items to load on all factors. Another difference between the two procedures is that the CFA provides goodness-of-fit indices of the hypothesized factor structure to the observed data and researchers typically use maximum likelihood to estimate factor loadings, whereas with the EFA Maximum Likelihood (ML) is only one of a variety of estimators used (Lawley \& Maxwell, 1971). Finally, the CFA allows the researchers to specify correlated measurement errors, constrain loadings or factor correlations to be equal to one another, perform statistical comparisons of alternative models, test second-order factor models, and statistically compare the factor structure of two or more groups.

Unlike EFA, which extracts factors from the data in the one way that maximizes the common or total variance explained, the CFA uses a pre-specified model to generate a predicted set of item interrelationships. The difference between each of these predicted interrelationships and the actual observed interrelationship is referred to as a fitted residual (Lawley $\&$ Maxwell, 1971) and is evaluated with a goodness-of-fit index. Thus in order to gauge how well a CFA model fits the data, a goodness-of-fit index is computed. A variety of different indices of relative fit have been developed.

Some of the most commonly used goodness-of-fit indices are the Tucker-Lewis coefficient (TLC), adjusted goodness-of-fit (AGFI), comparative fit index (CFI), incremental fit index (IFI), Normed fit index (NFI), and the Normed fit index (NFI). The Goodness-of-Fit Index (GFI) and the (AGFI) (Joreskog \& Sorbom, 1984) compare the ability of a model to reproduce the variance-covariance matrix. Specifically, the AGFI adjusts the GFI for the 
number of degrees of freedom expended in estimating model parameters. Bentler and Bonnett (1980) proposed a Normed Fit Index (NFI), which compares model fit to that of a model for the same data presuming independence of the measured or observed variables. Despite its wide use, the NFI has been shown to underestimate when the sample size is small. As a result, Bentler and Bonnett (1990) proposed the Comparative Fit Index (CFI), which takes sample size into account. Some researchers have suggested that the CFI should be the fit statistic of choice (Byrne, 1998). Another measure of goodness-of-fit is the Root Mean Square Residual (RMSR) that measures the average size of the residuals generated by the particular model and is used to compare the fit of two or more different models from the same data (Lawley $\&$ Maxwell, 1971). RMSR represents the absolute value of the average fitted residuals for a given specified model. As noted previously, fitted residuals are the difference between the actual correlations, or covariances, among the observed indicators and the correlations predicted by a particular model (Lawley \& Maxwell, 1971). Steiger and Lind (1980) focused on estimated population fit in their proposed root mean square error of approximation (RMSEA). The root mean-square residual (RMR) evaluates the average residual value for the variance-covariance matrix.

The various fit indices outlined above provide a collection of information about competing model being considered in SEM analyses. As some fit indices evaluate different aspects of fit, it is important to evaluate model fitness on multiple fit statistics as to ensure that judgments will not be an artifact of analytic choice (Byrne, 1998). Further, as Byrne (1998) emphasized, the assessment of model fit must be based on multiple criteria that take into account theoretical, statistical, and practical consideration. Overall, despite variations in the specific mathematical formulas, most of these comparative fit indices except the RMSR, RMSEA, and the RMR, basically reflect how well the given factor model fits the data and share a common feature of ranging between zero and one, with higher values indicating better fit. 
Regarding RMSR, RMSEA, and the RMR, the closer these values are to zero, the better the fit of the model. 


\section{SCALE DEVELOPMENT IN INTELLECTUAL DISABILITIES}

As mentioned previously, the assessment of individuals with ID presents unique challenges for the clinician and tends to rely heavily on behavior rating scales. However, the development of behavior rating scales follows the same general process as other measures of psychological functioning and is comprised of five primary steps: (1) test conceptualization; (2) test construction; (3) test tryout; (4) item analysis; and (5) test revision.

\section{Test Conceptualization}

The first step in the development of any measurement scale is test conceptualization. In this step, a researcher interested in a particular area of psychological functioning or construct decides that a test would be helpful or is needed in order to fully study the specific aspects of the area of interest. Part of this step is specifying the construct based on a synthesis of a series of impressions (Sternberg \& Grigorenko, 1997). It is up to the researcher, or test developer, to convince the test user that the construct being measured is a reasonable assimilation and synthesis of ideas (Kline, 2005). In the social sciences, arguments are commonplace about what a particular construct means as one person's definition may differ from another's.

When a scale is developed, it is expected that responses to items provide information that allow inferences to be made about the construct. A review of the literature will indicate whether the construct has been examined previously, if a test to measure it has already been developed and, if tests exist, how sound their psychometric properties are. Often at this point, the researcher will find that the construct has been examined and a new test is not needed. However, if the researcher does not like the available tests or one does not exist, they may decide that a new test is warranted. Preliminary questions test developers must consider are (Cohen \& Swerdlik, 1998): 
1. What is the test designed to measure?

2. What is the objective of the test?

3. Is there a need for the test?

4. Who will use the test?

5. Who will take the test?

6. What content will the test cover?

7. How will the test be administered?

8. What is the ideal format of the test?

9. Should more than one form of the test be developed?

10. What special training will be required for administering or interpreting the test?

11. What types of responses will be required by test takers?

12. Who benefits as the result of an administration of this test?

13. Is there any potential harm as the result of an administration of the test?

14. How will meaning be attributed to scores on the test?

\section{Test Construction}

When a researcher decides that the construct of interest has not been adequately measured or examined, the second step in the process is test construction. This step begins with scaling, or the process of setting rules for assigning numbers in measurement. During scaling, the researcher must decide what scale values will be assigned to different amounts of the trait, attribute, or characteristic being measured. Historically, L. L. Thurston is credited for being at the forefront of the efforts to develop methodologically sound scaling methods during his work to adapt psychophysical scaling methods to the study of psychological variables such as attitudes and values (Bock \& Jones, 1968; Thurston, 1959).

Test developers talk about different types of scales as a function of various characteristics. For example, scales can be categorized along a continuum of level of measurements. Scales can also be categorized in other ways such as the performance on a test as a function of age (i.e., age scale), or performance as a function of grade (i.e., grade scale). Further, a scale may be described in other ways such as one-dimensional versus multidimensional, or comparative versus categorical. Test developers must design a measurement method in a manner they believe is best suited to the way they have conceptualized the construct and how it should be measured. 
Essentially, a test taker is assumed to have more or less of a specific trait as a function of their test scores, that is, the higher or lower the score the more or less of the characteristic the individual possesses. There are several methods for determining the numbers assigned to different responses. However, researchers most often use rating scales to evaluate behavior in the field of ID. One of the most frequently used rating scales is the Scale developed by Rensis Likert in 1932 (Allen, 1957). The Likert-type scale consists of a series of declarative statements of which the subject is asked whether they agree or disagree with each statement and how strongly. This type of scale has been used by researchers for over 50 years and is explained by Likert (1932) in his article, "A Technique for the Measurement of Attitudes," in which he reported very satisfactory reliability data and that results from his scales compared favorably with those obtained by the Thurston Scale.

Once the scale of the test has been determined, the researcher must next develop the test item pool. The first step in item development regarding behavior rating scales for use with individuals with ID is to identify behaviors that are of particular concern to clinicians, parents, teachers, and caregivers. Typically, this can be done by using a survey of health practitioners, a review of literature on specific behavioral functions and their behavioral correlates or associated behaviors, behavior descriptions, and relevant association guidelines (i.e. American Association on Mental Retardation, American Psychological Association). This form of item generation will usually yield about twice as many items that will appear in the final version of the scale, but all are necessary in order to develop a test that is both reliable and valid.

\section{Test Tryout}

After the researcher has developed the initial item pool, the items must be tested. The testing should be conducted on individuals similar in critical respects to the individuals for whom the test is being developed. An important consideration in test tryout is how many 
individuals should be used in the initial tryout. Although there are no specific rules, some researchers have recommended that there be no fewer that five individuals per test item while some have suggested that ten individuals per item is preferable (Cohen \& Swerdlik, 1998). Generally, the more individuals involved in the tryout the better because it lessens the role of chance in subsequent statistical and factor analyses (Floyd \& Widaman, 1995).

In addition to trying out the test on individuals as similar as possible to the target individuals, the test should be tried out in conditions as similar as possible for to those for which it was designed. Thus, if a test is designed to be given by clinicians to care takers of individuals who reside in an inpatient facility, this is where the test should be tried out. Trying out the test under similar conditions allows the researcher to better evaluate items, examiners and examinee' reactions during the testing session, how well the test instructions match the situation, and how well the items are written to the particular conditions under which the test is to be used (Crocker \& Algina, 1986).

\section{Item Analysis}

The fourth step in test development after test tryout is item analysis. The process of item analysis is an important step because the goal of test construction is to develop a test of minimum length that yields scores with the necessary reliability and validity for its intended use. Although there are many statistical procedures that can be used to conduct an item analysis, many of these procedures are not relevant to the development of a behavior rating scale. The primary method used to develop behavior rating scales is the factor analysis.

The term factor analysis is used to describe a class of mathematical procedures designed to identify specific variables, or factors, that are typically attributes, characteristics, or dimensions on which people may differ. A factor analysis can be used to get both convergent and discriminatory evidence of construct validity (Cohen \& Swerdlik, 1998) and is conducted 
either on an exploratory or confirmatory basis. According to Floyd and Widaman (1995), an EFA typically entails "estimating, or extracting factors; deciding how many factors to retain; and rotating factors to an interpretable orientation" while in a CFA, "a factor structure is explicitly hypothesized and is tested for its fit with the observed covariance structure of the measured variables."

The factor analysis procedure is designed to determine what the common factors are that account for item variance. Essentially, the factor analysis is a measurement estimate of the correlation between items and the factors, these correlations are called factor loadings. When conducting a factor analysis, the researcher looks for those test items that correlate highly with the factor of interest. Typically, when examining the results of a factor analysis, the researcher looks for items with a factor loading of .80 or higher. As factor analysis procedures are highly complex and mathematically challenging, the procedure is a common component of standard computer-based statistical software packages, such as SPSS.

For example, if an item related to the factor of aggression has a loading of .0, the item is not correlated in any way with aggression and the item would not be retained for the next version of the test. However, if the item has a loading of .9 , then the item is highly correlated with aggression and would be retained as a test item. Once the researcher has identified those test items that load highly onto the factor, that is those items that account for the most variance, a CFA is conducted to examine how well the final test structure measures and represents the construct of interest. Results of the CFA indicate to the researcher if the test has enough items to adequately represent the factor and if the item scores yield the necessary information to be considered a reliable and valid measure of the construct being measured.

\section{Test Revision}

Typically during item analysis vast amounts of data are generated about the test items and the proposed test itself. However, as the primary method of item analysis for the 
development of behavior rating scales is the factor analysis, much of the data that can be generated will not be. In the case of behavior rating scales, test revision generally entails determining if the items chosen for inclusion adequately represent the construct of interest. Other issues regarding the chosen items are their readability (i.e., ease of reading and understanding for the test administrator); adequate factor loadings; specificity of instructions for administrators, raters, and for interpretation; and overall test utility. During this phase, researchers may want to re-examine their item pool and substitute items that may be more representative or rewrite items that may be difficult to understand. After revision, the researcher must return to step three and tryout the proposed test again. After tryout, another item analysis is conducted and data analyzed.

After the test has been revised and tried out for the second time, or more, the researcher may decide that the test is in its final form. At this point, the researcher can develop the test norms from the data and the test will be said to have been standardized on the second sample (Cohen \& Swerdlik, 1998). Standardization can be seen as the "process employed to introduce objectivity and uniformity into test administration, scoring, and interpretation" (Robertson, 1990). Essentially, the standardization sample represents the performance of the group of individuals against whom the examinees' performance will be compared. It is important that the standardized sample group is representative of the population on those variables that may affect test performance. Once the test items have been finalized and the test has been standardized, it is ready for the final step of cross-validation, the revalidation of a test on a different sample of test takers. 


\section{QUESTIONS ABOUT BEHAVIORAL FUNCTION (QABF)}

One of the few empirically derived and psychometrically sound behavior rating scales used in the brief functional assessment of behavior is the QABF, a 25-item questionnaire designed to rate specific behavioral functions and maintaining variables on a Likert-type scale of 0, never occurs, to 3, occurs often (Vollmer \& Matson, 1995).

\section{Scope and Development}

The QABF was developed in 1995 in response to the limitations inherent in the functional analysis procedure (Vollmer \& Matson, 1995). Questions in the QABF are designed to address and identify common behavioral functions of individuals with ID found in the research literature and clinical practice. Although the QABF is similar in structure to the Motivation Assessment Scale (MAS; Durand \& Crimmins, 1988), it addresses a higher number of behavioral functions and is comprised of five subscales: (1) attention; (2) escape; (3) nonsocial; (4) physical; and (5) tangible. Each behavioral function has five corresponding items on the scale that are rated by informants with respect to how often a behavior occurs in a particular context.

The development of the QABF followed the common test development procedures described previously. First, the researchers identified their construct of interest, specifically behavioral function. Second, a comprehensive literature review regarding behavioral function and expressions of communicative behavior was conducted to identify potential test items. Third, the items were piloted on a representative sample of individuals the test was being designed for. Fourth, the results of the test tryout, the item analysis, were examined using a factor analytic procedure. The factor analysis revealed and determined the five factors, or subscales, presently found in the QABF. Further analyses narrowed the item pool and the revised scale was tried out. The second administration yielded the finalized QABF-SF. 


\section{Psychometric Properties and Utility}

In 1996, Matson et al. presented the initial psychometric data for the QABF at the $22^{\text {nd }}$ Annual Convention of the Association for Behavior Analysis. The initial sample pool was comprised of 462 individuals living in a residential state training center for individuals with ID. Participants ranged in age from 13 to 86 years and predominantly functioned within the severe to profound range of ID. Behaviors examined during the initial tryout and evaluation of the QABF included typical behaviors exhibited by individuals with ID such as self injury, aggression, and property destruction. Both internal reliability and validity data were assessed. The initial psychometric analysis yielded coefficient alpha and Guttman split-half reliability coefficients of 0.86 and 0.91 , respectively. Further, an exploratory analysis with varimax rotation yielded the five factors seen in the final version with these factors accounting for $74.5 \%$ of the variance. The results of this study showed that the QABF could be a potentially useful tool in the brief functional assessment of challenging behavior in individuals with ID.

Although the initial psychometric data on the QABF was promising, further evaluation was needed. In 1999, Matson, Bamburg, Cherry and Paclawskyj conducted a validity study on the QABF examining the utility of the scale to predict treatment success for self injury, aggression, and stereotypies. The published study was comprised of two smaller studies with the first designed to establish the percentage of individuals whose maladaptive behavior could be ascribed to a clear behavioral function. In Experiment 1,398 individuals with moderate to profound ID from a developmental center for individuals with ID in Louisiana were administered the QABF.

For analysis purposes, participants were separated into three groups depending on their identified or target maladaptive behavior: (1) self injury ( $\mathrm{N}=118)$; (2) aggression ( $\mathrm{N}=83$ ); and(3) stereotypies ( $\mathrm{N}=197)$. In the self-injury group (Group 1), 78.3\% were Caucasian, $21.7 \%$ were 
African American, while 53.6\% were male and 46.4\% female; $6.6 \%$ of these individuals functioned within the severe range of ID while 93.4\% were within the profound range. Group 2, the aggression group, was comprised of 66.7\% Caucasians and 33.3\% African Americans; $56.8 \%$ were male while $43.2 \%$ were female. $3.9 \%$ of individuals in group 2 functioned within the severe range of ID while $96.1 \%$ functioned within the profound range. Lastly, Group 3 was comprised of $67.1 \%$ Caucasians and $32.9 \%$ African Americans with $72.7 \%$ being male and $27.3 \%$ female. Of this group, $4.5 \%$ were within the severe range while the remaining $95.5 \%$ within the profound range. Results of Experiment 1 indicated that the QABF could clearly identify behavioral functions, defined as subscales with a minimum score of 4 of 5 possible endorsements on a subscale with no other subscales containing significant endorsements, for $84 \%$ of the total sample (Matson et al., 1999). Specifically, the QABF was able to identify behavioral functions in 83\% of individuals in Group 1,74\% in Group 2, and 93.3\% in Group 3.

Experiment 2 in the Matson et al. (1999) study was designed to assess the utility of the QABF predictions of behavioral function for selecting effective treatments. In this study, 180 of the participants from Experiment 1 were selected at random and divided into a 30-member treatment group and a 30-member control group for each of the three behaviors examined in Experiment 1. Individuals in the treatment group had behavioral treatment plans driven by the QABF analysis and those in the control group had standard treatment protocols consisting primarily of interrupting, blocking, and redirection. Results of this study indicated that the behavior plans derived from the QABF significantly reduced the frequency of maladaptive behavior in the treatment group versus the control groups. Specifically, individuals in the self injury group experienced a $66 \%$ decline in the frequency of self injury in the treatment group versus $21 \%$ in the control group, a 59\% decrease in aggression was observed in the second treatment group versus 19\% in the aggression control group, and a 54\% decrease in stereotypies was seen in the treatment group versus a 15\% decrease in the control group. 
Also in 1999, Applegate, Matson, and Cherry used the QABF to evaluate the functional variables that affect severe problem behaviors in adults with ID. This study included 417 individuals with severe to profound ID who resided at a developmental training center in Louisiana who exhibited self-injury, stereotypy, aggression, pica, or rumination at least once every two weeks. Participants were separated into groups corresponding to their maladaptive behavior for the purposes of data collection and analysis. Results of this study showed that high frequency behaviors such as self injury, stereotypy, pica, and rumination seem to serve a predominantly non-social function whereas low frequency behaviors such as aggression are maintained by more externally maintained factors. These results further support the utility of the QABF for assessing behavioral function and allows for the identification of a number of behavioral antecedents within the five subscales.

In 2000, Paclawskyj, Matson, Rush, Smalls, and Vollmer conducted a two-part experiment to further examine the psychometric properties of the QABF. Specifically, this study was designed to look at the test-retest, inter-rater, and internal consistency of the QABF. In Experiment l, the authors included 34 individuals with severe and profound ID to examine the test-retest properties of the scale. Further, an additional 27 individuals were included to assess inter-rater reliability. Target behaviors of interest in this portion of the experiment were self injury, aggression, property destruction, tantrums/verbal aggression, stereotypy, pica, stealing, and elopement. To assess the stability of the individual QABF items over time, the authors conducted three measures of reliability: Spearman rank-order correlation coefficients; total agreement between items upon separate administration percentages; and Cohen's Kappa, where appropriate. Results showed that the Spearman rank-ordered correlations were high and ranged from .646 to 1.0 with $76 \%$ of items exceeding the minimal acceptable of 0.80 . Total percent agreement was found to be high with $96 \%$ of the items exceeding the minimum $80 \%$ and 
ranged from $69.57 \%$ to $95.95 \%$. Finally, Cohen's Kappa reliability coefficients ranged from .642 to 1.0 with $83 \%$ exceeding the minimum value of 0.70 .

To assess the stability over time for the QABF as a whole, the Pearson product-moment correlation coefficient was computed and found to be highly reliable (range $=0.795-0.990, p<$ .01). Inter-rater reliability of the QABF was determined by using the same statistics as for the test-retest analysis. Results of the Spearman rank-order correlation ranged from -0.095 to 1.0, with $52 \%$ of items exceeding the minimum of 0.80 ; thus, slightly lower than the teat-retest results. Total agreement was also slightly lower, ranging from $69.57 \%$ to $95.65 \%$, with $56 \%$ of items exceeding 80\% agreement. Cohen's Kappa values were also found to be slightly lower and ranged from $0.427-0.921$, with $41 \%$ of items exceeding a minimum of 0.70 . Finally, the Pearson product-moment correlations were found to be acceptable for each subscale and the total score (range $=0.79-0.987, p<.01)$.

Experiment 2 in this study included 243 additional participants to examine the internal reliability of the QABF and conduct a second EFA. Again, individuals in this sample functioned within the severe to profound range of ID. Target behaviors examined in this phase of the study included self injury, aggression, property destruction, tantrums/verbal aggression, stereotypy, pica, stealing, elopement, and rectal digging. To assess the internal consistency of the QABF, the authors calculated coefficient alpha for the individual subscales and the scale as a whole. Also, the Spearman-Brown statistic was calculated to assess the degree of consistency between halves of the test. Results of the coefficient alpha was very high for each subscale (range $=0.900-0.928$ ), but lower for the scale as a whole (0.601). The calculated Spearman-Brown statistic was found to be 0.600 . However, this low correlation was expected as the QABF was not designed to measure a homologous construct over the entire scale, but rather to measure a heterogeneous grouping of five functions of behavior. Results of the second EFA yielded five factors that 
corresponded to the subscales on the QABF and replicated the results of Matson et al. (1996). Therefore, the results of the two experiments in this study provided further support for the use of the QABF to identify possible behavioral functions in individuals with ID and the five subscale structure of the scale.

In an extension study of the QABF, Matson et al. (2001) examined the use of the QABF to identify the behavioral function of feeding problems in individuals with predominately profound ID. In this study, participants were 125 individuals at a residential developmental training center in Louisiana who displayed feeding problems such as food stealing, pica, rumination, food refusal, and other mealtime problem behaviors such as self injury and aggression. Participants were screened using the Screening Tool of fEeding Problems (STEP; Matson $\&$ Kuhn, 2001), a 25-item informant based measure that consists of 23 items and five subscales used for the identification of feeding problems among individuals with ID. Results of this study provide evidence that behavioral function varies across feeding problems and indicates that the QABF may also be useful in differentiating the function of feeding problems. Although this was a preliminary investigation and further research is needed to establish the diagnostic and treatment utility of the QABF to assess feeding problems, these results demonstrate that the QABF may be a useful tool in the analysis of other classes of behavior problems.

Other authors have also investigated the psychometric properties of the QABF in settings outside of the United States. Nicholson, Konstantinidi, and Furniss (2006) replicated and extended the findings of Matson and colleagues with regard to the psychometric properties of the QABF in a sample of 40 individuals in the north of England. Individuals in this study were 28 males and 12 females aged between 10 and 26 years residing in four residential schools for individuals with autism and/or severe learning disabilities and challenging behavior, In this 
study, the QABF was completed on 118 challenging behavior displayed by the individuals, the behaviors included aggression, property destruction, and self injury. Results of this study found that the levels of inter-rater reliability for both individual items and the scale as a whole were high, that inter-rater reliability of subscale scores was higher than those reported for other comparable rating scales. The authors also found that the internal consistency was high for all subscales of the QABF and for the scale as a whole. Finally, the results of the factor analysis yielded five factors that corresponded clearly with those on the QABF.

Recently, Singh et al. (2006) adapted the QABF for use with individuals with serious mental illness who engage in maladaptive behavior, and assessed the psychometric characteristics of the new scale (Questions About Behavioral Function in Mental Illness; QABFMI). In this study, the authors evaluated a sample of 135 adults with serious mental illness from three inpatient psychiatric hospitals. Results of factor analyses provided a conceptually meaningful five-factor solution: physical discomfort; social attention; tangible reinforcement; escape; and non-social reinforcement. Congruence between the five factors derived with the QABF-MI and the corresponding factors in the original QABF was perfect. The authors also assessed the reliability of the QABF-MI. The inter-rater reliability ranged from .96 to .98 and Pearson $r$ test-retest reliability ranged from .86 to .99. Further, Coefficient alpha ranged from .84 and .92 , thus indicating substantial internal consistency of each of the five factors. The results of this study indicate that the QABF-MI has robust psychometric properties and may be useful as a screening tool for determining the nature of the variables that maintain maladaptive behavior exhibited by individuals with serious mental illness. Although further examination is needed, these results provide further support for the use of the QABF to evaluate the behavioral function of individuals and extend its use to the area of mental illness.

Based on a review of the literature, the psychometric properties of the QABF have been well established and replicated several times across different settings with similar results. It 
appears that the QABF is a highly reliable and valid instrument for the assessment and identification of behavioral function in individuals with a range of ID and a variety of challenging behaviors. There appears to be no other scale in the literature that assesses behavioral function that is as psychometrically sound, valid, reliable, or clinically useful. 


\section{PURPOSE}

Given the lack of time and resources faced by most professionals in clinical settings discussed previously, it is imperative that assessment measures be as brief as possible. Briefer assessments are also desirable when a measure forms only one part of a battery of assessments, and the overall time demands on the clinician can easily become burdensome. However, briefer measures must be as, if not more, valid and reliable than the original and provide the clinician with the necessary information and data to aide in assessment and treatment selection.

Obviously, this is a difficult balance to reach. Researchers have clearly demonstrated the utility of brief functional assessment measures to achieve these tasks primarily using the QABF. Thus, the purpose of this study was to attempt to develop a short form of the QABF with high validity and reliability while maintaining the intent of the measure to accurately and reliably identify the function of problematic behavior. 


\section{METHOD}

\section{Participants}

Data for this study was obtained from 589 individuals with varying degrees of ID and maladaptive behavior problems who reside at Pinecrest Developmental Center (PDC), a residential training center for individuals with ID in Louisiana. Specifically, data consisted of demographic information and raw QABF scores obtained during routine annual clinical assessments. Individuals in this sample were between the ages of 19 and $85(M=47.31, S D=$ 13.54), of whom 319 (54.2\%) were male and 270 (45.8\%) female. There were $466(79.1 \%)$ Caucasians, 121 (20.5\%) African Americans, and 2 (0.3\%) Hispanics. Six (1.0\%) individuals were diagnosed with mild mental retardation, 16 (2.7\%) moderate, 67 (11.4\%) severe, and 500 (84.9\%) diagnosed with profound mental retardation. QABF data from these individuals were randomly divided using SPSS to obtain two samples on which to conduct exploratory and confirmatory factor analyses.

\section{QABF Exploratory Factor Analysis}

After randomly selecting approximately 50\% of the total sample, QABF data from 304 individuals were used to conduct an EFA utilizing the PAF method of extraction. This sample size was sufficient to meet and exceed the subject-to-item ratio of five to one typically viewed as necessary for deriving a suitable factor solution (Arrindell \& van der Ende, 1985; Kass \& Tinsley, 1979). The subject-to-item ratio for this EFA was just over twelve to one. The EFA explored the structure of the components and item loadings of the original QABF. This sample consisted of 168 (55.3\%) males and 136 (44.7\%) females of which 241 (79.3\%) were Caucasian, 61 (20.1\%) African American and 2 (0.7\%) Hispanic. These individuals ranged in age from 20 to 85 (M = 47.51, SD = 14.33). Four (1.3\%) individuals were diagnosed with mild mental retardation, 11 (3.6) moderate, 29 (9.5\%) severe, and 260 (85.5\%) profound. 


\section{Item Selection}

The goals of the item selection procedure were to reduce the length of the QABF while, (a) preserving the content of all five factors measured by the QABF, (b) retaining a minimum of three items per scale, (c) maintaining significant reliability estimates, (d) providing a factor structure in which goodness-of-fit indices met acceptable standards, and (e) retaining the original context of each of the five QABF factors. In order to meet these goals in the selection of items, the following guidelines were used:

1. Items that best measure the intended construct as inferred by the size of its factor loading on the EFA and its respective corrected item-total correlation.

2. Items that have minimal cross-loadings.

3. Items that have minimal correlated uniqueness, particularly with regard to other items in the same subscale. In cases where two items within the same subscale had significant correlated uniqueness, only one of the two items was retained.

4. Items that met a subjective evaluation of content and clinical relevance. For example, small drops in scale reliability were sometimes sacrificed if a different item set provided greater practical use or predictive power. The items were scrutinized by a group of 10 behavior analysts with experience in working with this population. Cronbach's alpha was calculated for each item and agreement regarding inclusion or exclusion and was generally high with item coefficient alpha values ranging between .9763 and .7855 .

5. Sufficient items in each subscale to maintain a sufficient coefficient alpha estimate of reliability.

Data gathered during the item selection process allowed for an a priori calculation of the validity and reliability of the proposed short form. Initial analyses showed that the QABF-SF 
was expected to maintain the five factor structure and high reliability of the original measure. Mean internal consistency reliability estimates were calculated across the 5-item subscales of the original QABF, as was a Cronbach's alpha estimate for each of the 3-item subscales and the QABF-SF as a whole.

\section{QABF Confirmatory Factor Analysis}

QABF data from the remaining 285 individuals were used to conduct a CFA using EQS 6.1 to determine whether the factor structure required modification. The subject-to-item ratio used to conduct the CFA was above the five to one ratio suggested; the subject to item ratio in this analysis was just over eleven to one. The CFA was conducted to confirm the factor structure indentified by the EFA and, if possible, refine the model using a separate sample of participants. This sample was comprised of 151 (53.0\%) males and 134 (47.0\%) females who were 78.9\% (225) Caucasian and 21.1\% (60) African American. Of these individuals, 2 (0.7\%) were diagnosed with mild mental retardation, 5 (1.8\%) moderate, 38 (13.3\%) severe and 240 $(84.2 \%)$ profound. These individuals ranged in age from 19 to $82(M=47.10, S D=12.65)$.

In order to identify items to be considered for exclusion, the LaGrange multiplier was used. The LeGrange multiplier is a mathematical modification index within EQS 6.1 used to identify variables that worsen model fit. After items were identified for removal, another CFA was conducted and the goodness-of-fit indices examined. The following goodness-of-fit indices were used to assess the degree of fit between the proposed model and the sample data: $\chi^{2}$, Bentler-Bonett Normed Fit Index (NFI), Comparative Fit Index (CFI), Standardized Root Mean-Square Residual (RMR), and Room Mean-Square Error of Approximation (RMSEA).

\section{Short Form Development and Analyses}

After items were selected according to the guidelines outlined previously, 75 individuals were chosen for test tryout; these individuals were randomly selected from the 175 individuals 
who currently receive an annual QABF at PDC and who were not included in the original 589 participants used to analyze the QABF and develop the short form. These 75 individuals ranged in age from 19 to $77(M=46.33, S D=12.47)$. Thirty-seven $(49.3 \%)$ were male and $38(50.7 \%)$ female while 60 (80\%) were Caucasian and 15 (20\%) African American. Two (2.7\%) of these individuals were diagnosed with moderate mental retardation, 9 (12\%) severe, and 64 (85.3\%) profound.

Following the above discussed item selection guidelines, ten items were identified for elimination. Following test construction procedures, the shortened version of the QABF was administered to the group of 75 randomly chosen individuals described previously. The number of participants selected for participation in the QABF tryout is in accordance with the suggested subject-to-item ratio of five to one (Arrindell \& van der Ende, 1985; Kass \& Tinsley, 1979). Data from the tryout administration were examined using the multivariate statistical software EQS 6.l in order to examine the goodness-of-fit properties of the data as they relate to the proposed five factor model and obtain validity and reliability estimates of the short form version of the QABF. 


\section{RESULTS}

\section{QABF Exploratory Factor Analysis}

In order to examine the constructs that the QABF is purported to measure and explore item-fit within these constructs an EFA using SPSS 11.5 was undertaken on data from 304 individuals randomly selected from the 589 participants in this study. A principle component procedure utilizing varimax rotation was used to analyze the original 25-items on the QABF. This analysis extracted five factors with eigenvalues greater than 1.0 that accounted for $73.9 \%$ of the total item variance. Eigenvalues for each of the components were 6.717, 3.705, 3.277, 2.498, and 2.272, respectively. Table 1 presents the rotated sums of squares loadings and percentage of variance explained by each component.

Table 1. EFA Rotated Sums of Squares Loadings $(\mathrm{N}=304)$.

\begin{tabular}{ccc}
\hline Component & \% of Variance Explained & Cumulative \% of Variance \\
\hline 1 & 15.78 & 15.78 \\
3 & 15.33 & 31.11 \\
4 & 14.65 & 45.76 \\
5 & 14.10 & 59.86 \\
\hline
\end{tabular}

A summary of the rotated principle components and item loadings appears in Table 2. These results are highly consistent with other exploratory investigations of the psychometric properties of the QABF (Matson et al., 1996; Paclawskyj et al., 2000; Nicholson, 2006; Singh et al., 2006). 
Table 2. EFA Principle Components and Item Loadings of the 25-item QABF ( $\mathrm{N}=304)$.

\begin{tabular}{|c|c|c|c|c|c|}
\hline \multirow{2}{*}{ Item } & \multicolumn{5}{|c|}{ Component } \\
\hline & 1 & 2 & 3 & 4 & 5 \\
\hline 24 & .895 & .045 & .074 & -.046 & .010 \\
\hline 14 & .892 & .087 & .027 & -.010 & .024 \\
\hline 19 & .890 & .089 & .032 & .003 & -.025 \\
\hline 4 & .888 & .051 & .103 & -.074 & .037 \\
\hline 9 & .816 & .013 & .033 & -.056 & .087 \\
\hline 22 & .088 & .867 & .009 & -.183 & .026 \\
\hline 17 & .135 & .859 & -.028 & .175 & .012 \\
\hline 7 & .044 & .852 & .050 & -.098 & .120 \\
\hline 12 & .051 & 849 & .119. & -.165 & .145 \\
\hline 2 & -.015 & .800 & .091 & -.049 & 193 \\
\hline 21 & .118 & -.002 & .892 & -.094 & .128 \\
\hline 11 & .096 & .093 & .882 & -131 & .090 \\
\hline 16 & .072 & .047 & .868 & -.010 & 102 \\
\hline 1 & .095 & .074 & .834 & -196 & .162 \\
\hline 6 & -.070 & .030 & .705 & .056 & .046 \\
\hline 23 & -.083 & -199 & -.110 & .859 & -.127 \\
\hline 3 & -.029 & -209 & -.035 & .817 & -.157 \\
\hline 8 & .053 & -.163 & -.132 & .793 & -.162 \\
\hline 18 & -.025 & -.014 & -.080 & .788 & -.070 \\
\hline
\end{tabular}




$\begin{array}{llllll}13 & -.100 & -.098 & .022 & .767 & -.131 \\ 15 & .040 & .076 & .114 & -.101 & .871 \\ 25 & .007 & .074 & .052 & -.191 & .867 \\ 5 & .019 & .093 & .066 & -.187 & .832 \\ 20 & .018 & .014 & .144 & -.128 & .775 \\ 10 & .060 & .280 & .147 & -.036 & .682\end{array}$

To illustrate the similarities between extraction methods, specifically PCA and Principle Axis Factoring, a second EFA was conducted using the Principal Axis Factor procedure. This second analysis also extracted five factors with eigenvalues greater than 1.0. These factors accounted for $67.7 \%$ of the total item variance and eigenvalues for each of the components were $6.407,3.430,2.986,2.182$, and 1.920 , respectively. Table 3 presents the rotated sums of squares loadings and percentage of variance explained by each component.

Table 3. EFA Rotated Sums of Squares Loadings $(\mathrm{N}=304)$.

\begin{tabular}{ccc}
\hline Component & \% of Variance Explained & Cumulative \% of Variance \\
\hline 1 & 14.71 & 14.71 \\
2 & 14.13 & 28.84 \\
3 & 13.52 & 42.35 \\
4 & 12.69 & 55.04 \\
5 & 12.66 & 67.70 \\
\hline
\end{tabular}

Table 4 presents the rotated principle components using the Principal Axis Factor method of extraction. These results are highly consistent with those using PCA extraction. 
Table 4. EFA Principle Axis Factoring and Item Loadings of the 25-item QABF ( $N=304)$.

\begin{tabular}{|c|c|c|c|c|c|}
\hline \multirow{2}{*}{ Item } & \multicolumn{5}{|c|}{ Component } \\
\hline & 1 & 2 & 3 & 4 & 5 \\
\hline 24 & .873 & .046 & .073 & -.048 & .012 \\
\hline 14 & .867 & .087 & .028 & -.012 & .025 \\
\hline 19 & .866 & .089 & .033 & .001 & -.022 \\
\hline 4 & .866 & .052 & 102 & -.074 & .038 \\
\hline 9 & .753 & .021 & .039 & -.059 & .081 \\
\hline 22 & .088 & .842 & .011 & -.186 & .031 \\
\hline 17 & .133 & .831 & -.025 & -.177 & .019 \\
\hline 12 & .052 & .822 & .120 & -169 & .147 \\
\hline 7 & .047 & .811 & .050 & -.107 & .124 \\
\hline 2 & -.007 & .740 & .090 & -.065 & .192 \\
\hline 21 & .115 & .000 & .887 & -093 & .128 \\
\hline 11 & .095 & .093 & .870 & -.129 & .092 \\
\hline 16 & .072 & .048 & .828 & -.013 & .106 \\
\hline 1 & .094 & .076 & .828 & -194 & .163 \\
\hline 6 & -.050 & .028 & .596 & .034 & .059 \\
\hline 23 & -.082 & -194 & -108 & .860 & -.126 \\
\hline 3 & -.031 & -205 & -.037 & .787 & -160 \\
\hline 8 & .045 & -164 & -.128 & .745 & -167 \\
\hline 18 & -.028 & -.034 & -.080 & .703 & -.086 \\
\hline
\end{tabular}




$\begin{array}{llllll}13 & -.096 & -.110 & .009 & .687 & -.140 \\ 25 & .007 & .077 & .059 & -.191 & .849 \\ 15 & .040 & .081 & .119 & -.102 & .849 \\ 5 & .021 & .098 & .074 & -.189 & .793 \\ 20 & .019 & .031 & .150 & -.135 & .696 \\ 10 & .059 & .264 & .149 & -.066 & .602\end{array}$

To illustrate the significance of the rotational procedure after the extraction is performed, an unrotated analysis was conducted. The results of the unrotated percentages of explained variances are presented in Table 5.

Table 5. EFA Eigenvalues and Unrotated Sums of Squares Loadings ( $\mathrm{N}=304)$.

\begin{tabular}{cccc}
\hline Component & Eigenvalues & \% of Variance Explained & Cumulative \% of Variance \\
\hline 1 & 6.717 & 26.87 & 26.87 \\
2 & 3.705 & 14.82 & 41.69 \\
3 & 3.277 & 13.11 & 54.80 \\
4 & 2.498 & 9.99 & 64.79 \\
5 & 2.272 & 9.09 & 73.88 \\
\hline
\end{tabular}

\section{Item Selection}

After following the item selection procedure outlined previously, 10 items were identified for removal. The result was a 15-item version of the QABF. Items selected for retention are presented in Table 6 while items not selected for retention appear in Table 7. 
Table 6. Retained Items for the Short Form.

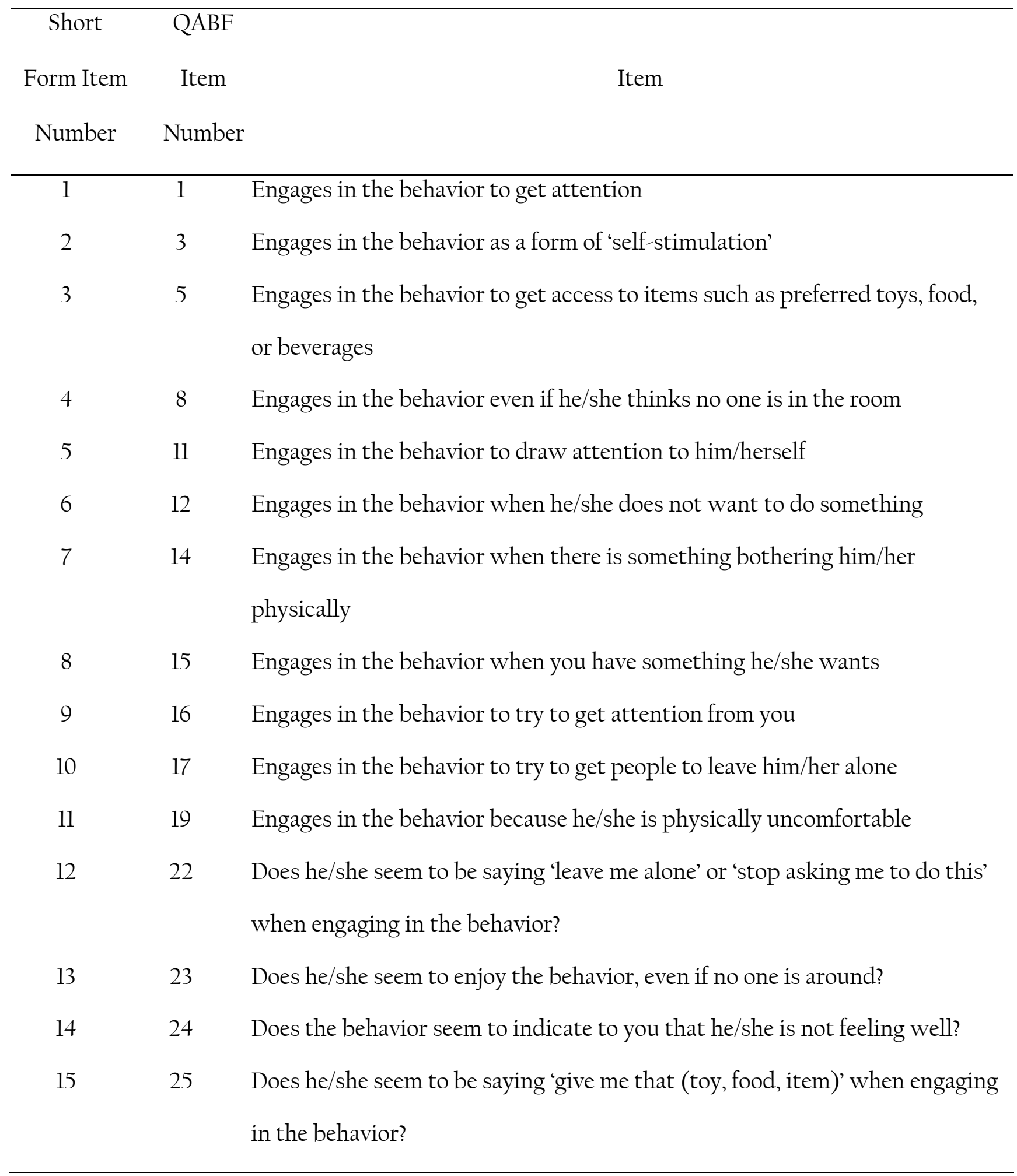


Table 7. Items Not Retained for the Short Form.

\begin{tabular}{cl}
\hline $\begin{array}{c}\text { QABF Item } \\
\text { Number }\end{array}$ & \\
\hline 2 & Engages in the behavior to escape work or learning situations \\
4 & Engages in the behavior because he/she is in pain \\
6 & Engages in the behavior because he/she likes to be reprimanded \\
7 & Engages in the behavior when asked to do something (get dressed, brush teeth, \\
9 & Engrk, etc.) \\
10 & Engages in the behavior when you take something away from him/her \\
13 & Engages in the behavior because there is nothing else to do \\
& Engages in the behavior in a highly repetitive manner, ignoring his/her \\
20 & surroundings \\
21 & Engages in the behavior when a peer has something he/she wants \\
& behavior?
\end{tabular}

As noted earlier, the item selection process allowed for an a priori calculation of the validity and reliability of the proposed short form. Analyses showed that the QABF-SF was expected to maintain the five factor structure and high reliability of the original measure. With a mean internal consistency reliability estimate of $\alpha=0.914$ across the 5 -item subscales of the original QABF, a Cronbach's alpha estimate predicted the 3-item subscales would show a mean internal consistency of $\alpha=0.905$. Slight reductions in internal consistency are not necessarily 
problematic when the measure is designed to assess a broad domain using few items. Boyle (1991) recommends modest reliabilities of between $\alpha=0.65$ to 0.75 when the construct being measured is broad. As the short form was composed of items that showed high factor loadings and not items chosen at random, it was expected that internal consistency reliability estimates of the subscales of the 15-item QABF-SF be similar to those of the original QABF.

\section{QABF Confirmatory Factor Analysis}

To further explore and cross validate the five factor structure of the QABF, a CFA was undertaken on data from the 285 participants not selected for use in the EFA using EQS 6.1. Then, the LaGrange multiplier was used to identify items for exclusion to improve goodness-offit and another CFA was run. This procedure was conducted twice in an attempt to examine possible modifications by removing items that worsen model fit and thus improve goodness-offit indices. Fit indices for each of the models tested are presented in Table 7. The final model shows an excellent fit between the model and the data, where $\chi^{2}(d f=90)=200.547, \mathrm{NFI}=.921$, $\mathrm{CFI}=.955, \mathrm{RMR}=.167$, and $\mathrm{RMSEA}=.066$.

Table 8. Fit Indices for Each Model Tested for the CFA $(\mathrm{N}=285)$.

\begin{tabular}{lccccccc}
\hline Model Step & $\chi^{2}$ & $d f$ & $p$ & NFI & CFI & RMR & RMSEA \\
\hline Initial model & 576.447 & 275 & .000 & .879 & .933 & .150 & .062 \\
Items 6, 2, 13, 9, and 10 removed & 360.219 & 170 & .000 & .906 & .948 & .153 & .063 \\
Items 21, 7, 18, 4, and 20 removed & 200.547 & 90 & .000 & .921 & .955 & .167 & .066 \\
\hline
\end{tabular}

\section{QABF-Short Form (QABF-SF) Tryout}

Following test construction procedure, the 15-item QABF-SF was tested. The QABF-SF was administered by trained researchers to frontline staff regarding the problem behaviors of 75 
randomly selected individuals from the 175 individuals who are currently administered a QABF on an annual basis. The number of participants in this step met the suggested subject-to-item ratio of five to one (Arrindell \& van der Ende, 1985; Kass \& Tinsley, 1979).

\section{QABF-SF Analysis}

To examine the goodness-of-fit properties of the data as they relate to the proposed five factor model and obtain validity, and reliability of the short form version of the QABF, a CFA was conducted using EQS 6.1.

Goodness-of-Fit

Consistent with current practice, the Maximum-Likelihood (ML) estimation procedure was used to determine goodness-of-fit properties of the 5-factor model of the QABF-SF using data from all 75 participants of the tryout. Parameter characteristics and change indices of the proposed short form were evaluated for model and criterion validity using EQS 6.1. Estimations of model fit were high $\chi^{2}(80)=154.103, p<.01$, Bentler-Bonett Normed Fit Index $(\mathrm{NFI})=.824$, Comparative Fit Index $(\mathrm{CFI})=.904$, while estimates of error were fairly low, Standardized Root Mean-Square Residual $($ RMR) =.091, and Root Mean-Square Error of Approximation (RMSEA) $=.112$. Table 8 presents the model fit indices for the original, 15 -item model and shortened forms of the QABF.

Table 9. Fit Indices for the QABF and QABF-SF

\begin{tabular}{lccccccc}
\hline Model & $\chi^{2}$ & $d f$ & $p$ & NFI & CFI & RMR & RMSEA \\
\hline Original QABF & 576.447 & 275 & .000 & .879 & .933 & .150 & .062 \\
15-Item Model & 200.547 & 90 & .000 & .921 & .955 & .167 & .066 \\
QABF-SF & 154.103 & 80 & .000 & .842 & .904 & .091 & .112 \\
\hline
\end{tabular}


Figure 1 presents the five factor solution and item loadings of the QABF-SF. These five factors were labeled, as on the original QABF: Factor 1, Attention; Factor 2, Escape; Factor 3, Non-Social; Factor 4, Physical; and Factor 5, Tangible. Each of the items in Factor 1 (Attention) focused on attention seeking as the motivation for the target behavior. The three items in Factor 2 (Escape) all related to a desire to refrain from some activity. Factor 3 (Non-Social Reinforcement) items were all related to a tendency to engage in a target behavior in the absence of social reward, thus measuring a tendency to use a target behavior to seek stimulation and/or avoid boredom. Factor 4 (Physical Discomfort) items focused on illness or physical discomfort as the motivation for target behavior. Factor 5 (Tangible Reinforcement) items were all related to seeking a tangible item such as food or drinks or toys.

$\underline{\text { Reliability }}$

\section{Internal Consistency}

To assess the internal consistency of the QABF-SF, coefficient alpha for each subscale and the test as a whole was computed using data from all 75 of the individuals who participated in the QABF-SF tryout. The values of Cronbach's alpha calculated for each subscale were generally high. Subscale coefficient alpha values were .9243 for attention, .9103 for escape, .8351 for non-social, .9383 for physical, and .7907 for tangible. Alpha for the QABF-SF as a whole was somewhat lower and calculated as .5888.

Test-Retest

The test-retest properties of the QABF-SF were examined in 29 randomly chosen individuals from the pool of 75 who participated in the evaluation of the QABF-SF. Retest data for these individuals was collected with the same respondent two weeks after the initial administration of the QABF-SF. Pearson Product Moment Correlations for subscale scores were .980 for attention, .977 for escape, .986 for non-social, .952 for physical, and .836 for tangible. 


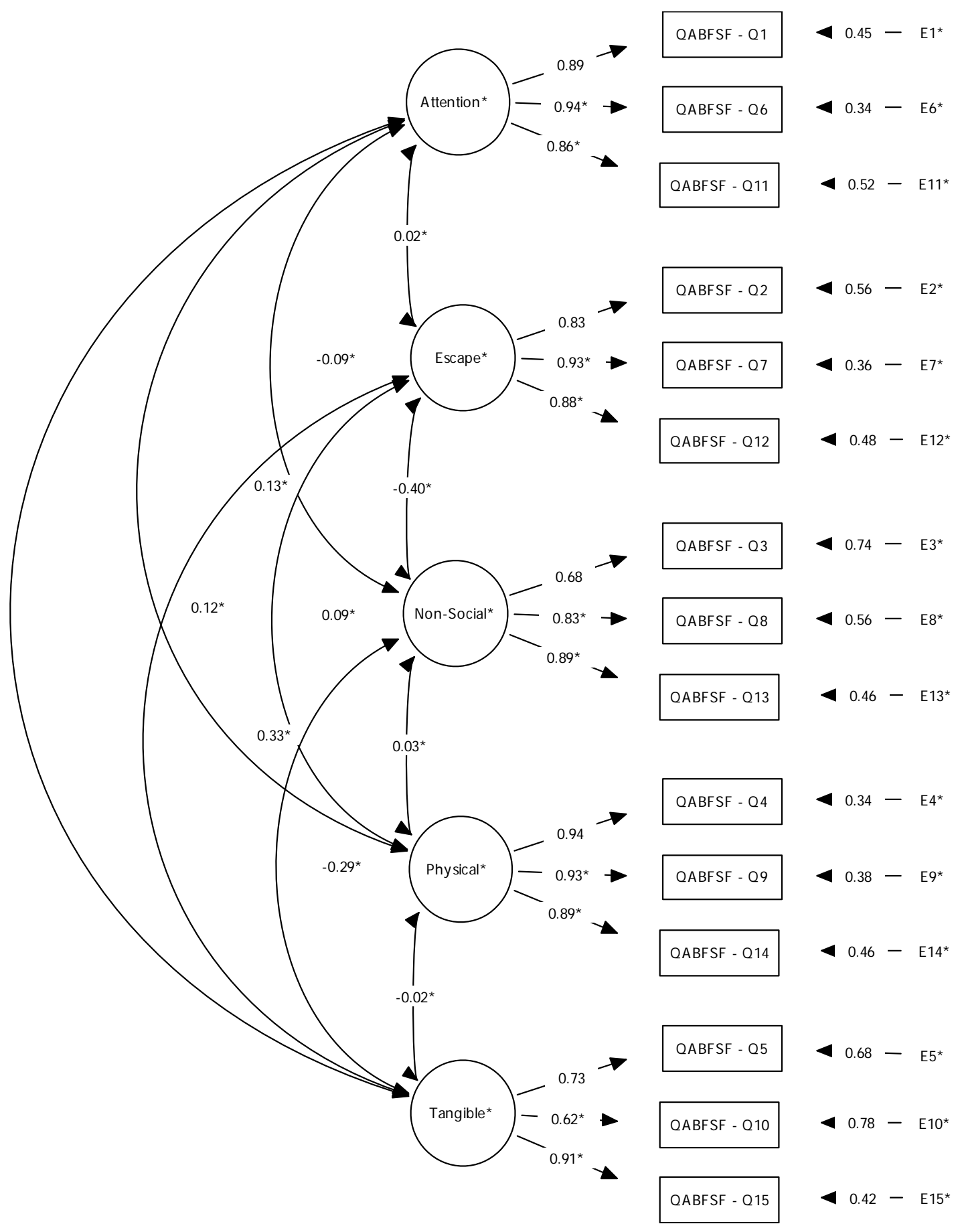

Figure 1. QABF-SF Confirmatory Factor Analysis $(\mathrm{N}=75)$. 
All of these correlations were significant at the 0.01 level. Spearman Rank Order Correlations between the sets of subscale scores provided by the same rater two weeks apart were calculated for each subscale were .966 for attention, .949 for escape, .972 for non-social, .952 for physical, and .836 for tangible. All of these correlation coefficients were significant at the 0.01 level.

Interrater

Interrater properties of the QABF-SF were calculated using the same correlation coefficients used to examine test-retest reliability. Data from two respondents regarding the function of maladaptive behavior in 38 individuals were analyzed. Subscale Pearson correlations were .932 for attention, .933 for escape, .955 for non-social, .927 for physical, and .815 for tangible. With respect to Spearman correlations, subscale scores were .792 for attention, .870 for escape, .909 for non-social, .840 for physical, and .815 for tangible. These correlation coefficients were all significant at the 0.01 level.

$\underline{\text { Social Validity }}$

An analysis of items designed to measure social validity showed that frontline staff liked the format, seemed to think that the QABF-SF could provide the treatment team with good information, and be useful as part of a brief functional assessment. Questions used to assess social validity and response data are presented in Table 10.

Table 10. Social Validity Questions and Responses $(\mathrm{N}=142)$.

\begin{tabular}{llc}
\hline & Yes & No \\
\hline $\begin{array}{l}\text { 1. Do you like the format of the QABF-SF? } \\
\text { (length and time of administration) }\end{array}$ & 128 & 14 \\
\hline
\end{tabular}

(table continued) 
2. Do you think that the QABF-SF provides the team with good information?

3. Do you think that the QABF-SF could be useful as part of a brief

functional assessment? 


\section{DISCUSSION}

Given increasing demands on clinicians to do more with fewer resources, a need for efficient methods of assessment has emerged. This need is pervasive throughout the entire healthcare industry and has significantly impacted the way that we take care of people. Within the field of behavioral psychology in ID, the need is for brief functional behavior assessment tools. Researchers and practitioners have acknowledged that the costs of conducting lengthy functional analog assessments characterized by applied behavior analysis far outweigh any potential benefits. As such, brief assessment methods have been developed for use by clinicians in settings outside the research laboratory. The literature regarding the use of brief functional behavior assessment shows that these assessments provide as much reliable and valid information regarding the maintaining consequences of a behavior as long and sometimes ethically questionable analog assessments.

The QABF (Vollmer \& Matson, 1995) is a rating scale that is extensively used in the behavior analytic literature as a method of developing hypotheses for the functions of maladaptive behavior in individuals with intellectual disability. In several studies, it has proven to have fairly robust psychometric properties, including reliability and validity. The present study was designed to determine if a shorter form of this rating scale (i.e., QABF-SF) would have similar psychometric properties as the original QABF.

The first part of this study examined the five constructs, or behavioral functions, that the QABF is purported to measure and explored item-fit within these constructs. An EFA was employed to examine the underlying factor structure of the QABF. Given the rather large sample pool, it was feasible in this study to randomly select approximately 50\% of the data for this purpose. The EFA procedure is designed to find the one underlying factor model that bests fits the data. To do this, the researcher must make several important decisions including 
choosing the method of extraction, number of factors to retain, type of rotation, and sample size. In this study, two different methods of extraction were used to illustrate the similarities between methods. Theoretically, if the factor structure is strong, that is if several items are highly correlated and seem to measure the same attribute, then the method of extraction will not produce differing results. Conversely, if the factor structure is weak and the items of interest seem to be measuring different attributes then the method of extraction will yield different results. In this study, the factor structure held across extraction methods.

As discussed previously, the most common method of deciding how many factors to retain is to examine the eigenvalues and keep those factors with an eigenvalue over 1.0. Although statisticians argue that this method is not as accurate as others, it is the most widely employed method for deciding how many factors to retain. The initial EFA conducted on the QABF supported a five factor solution of the 25 items, with 5 items loading on each factor. The next decision concerns the type of rotation method. The purpose of rotation is to mathematically maximize high loadings while minimizing low loadings to yield the simplest and, therefore, the most interpretable factor structure. In essence, the rotation procedure simplifies the output of the analysis and facilities the interpretation of the factor loadings.

The most common type of rotation method employed by statistical packages is the orthogonal method as conventional wisdom advises researchers to use this rotation method because it produces more easily interpretable results (Costello \& Osborne, 2005). In this study, the result of the orthogonal rotation was compared to the unrotated result to highlight the purpose and function of the rotation procedure. Results show that the percentage of variance in the unrotated solution was more variable than in the rotated solution. Thus, although the rotated solution explained the same overall percentage of variance in the data as the unrotated solution, the rotated solution affected the percentage of variance explained by each factor given 
that the goal of the rotation procedure is to mathematically clarify and simplify the factor structure. Therefore, although each factor may not have been equally represented in the data, as evident by the percentage of variance explained by each factor in the unrotated solution, rotating the factor solution clarified the nature of the factor structure and the factor loadings. The last decision the researcher has to make concerns sample size. The number of participants in each stage of this study was sufficient to meet or exceed recommendations found in the literature and was not a concern.

Data generated during the EFA of the QABF was used in the subsequent item selection procedure. The goals of the item selection procedure were to preserve the content of all five factors measured by the QABF, retain a minimum of three items per scale, maintain significant reliability estimates, provide a factor structure in which goodness-of-fit indices met acceptable standards, and retain the original context of each of the five QABF factors. After examining the results of the EFA, ten items were selected for removal, with the remaining items forming the QABF-SF.

In order to examine the item fit of the 25-item QABF, cross validate its five factor structure, and determine if any model modifications were necessary, a CFA was undertaken with the remaining data not used in the EFA. Although the EFA and CFA are both used to investigate the theoretical constructs that might be represented by the data, the CFA is used to evaluate how well the data fits into a predetermined factor model. Thus, while the EFA is used to build a theory, the CFA is used to test a theory. To test model fitness, the CFA procedure incorporates several goodness-of-fit indices that, although mathematically different, basically all reflect how well the given factor model fits the data with higher values indicating better fit.

The results of the initial CFA indicated that the original five factor model of the QABF fit well with the data as all fit indices were within acceptable limits. After utilizing the LaGrange 
multiplier designed to mathematically identify items that may worsen model fit, five items, one per factor, were selected for removal and another CFA was undertaken. The results of this second CFA indicated that removing five items slightly improved model fitness across all goodness-of-fit indices. Subsequently, the 20 remaining items were subject to the LaGrange multiplier in an effort to further improve model fit. Results of the second LaGrange multiplier procedure suggested five more items, one per factor, which could be eliminated in order to improve model fitness. After these items were removed, the 15 remaining items were subjected to a third CFA in order to examine model fitness. The results of this third CFA showed that removal of the five suggested items also improved model fitness as goodness-of-fit indices were all improved. Given that a factor becomes unstable with fewer than three items, no further model fitness analyses were conducted.

The second part of this study examined the factor structure, goodness-of-fit, reliability, and validity of the 15-item QABF-SF. To examine the model fit of the QABF-SF a CFA was conducted on the data gathered during the QABF-SF tryout. The results of this CFA indicated that the five factor structure and item loadings of the QABF-SF were consistent with those of the original QABF. Goodness-of-fit indices of the original QABF were all within acceptable limits and showed good model fit with the data. Results of the CFA on the original QABF after ten items had been removed during the model modification process showed excellent model fit with $\chi^{2}(90)=200.547, p<.01$, Bentler-Bonett Normed Fit Index (NFI) =.921, Comparative Fit Index $(\mathrm{CFI})=.955$, while estimates of error were fairly low with the Standardized Root MeanSquare Residual $($ RMR $)=.167$, and Room Mean-Square Error of Approximation (RMSEA) $=$ .066. Results of the CFA conducted on the QABF-SF also showed good model fit, although some of the indices were lower than expected. Specifically model fit indices were, $\chi^{2}(80)=154.103, p$ < .01 , the NFI $=.824, \mathrm{CFI}=.904, \mathrm{RMR}=.091$, and $\mathrm{RMSEA}=.112$. Although these values were lower 
than estimated during the model modification process, they were all still within acceptable ranges and show that the model fit of the QABF-SF is highly comparable to the initial model of the QABF.

Overall, results of the CFA on the QABF-SF showed that the short form retained the five factor structure of the original measure and that these five factors accurately reflected the data regarding the function of maladaptive behavior exhibited by individuals with ID. Hence, attention, escape, non-social reinforcement, physical discomfort, and access to a tangible can be interpreted as distinct functional aspects of an individual's behavior. Thus, the QABF-SF retains the intent of the QABF, to accurately identify the function of maladaptive behavior among individuals with ID.

Data from the CFA were also used to examine the reliability and validity of the QABFSF. Results showed that, although the internal consistency reliability of the QABF-SF was not as high as estimated a priori, with a mean $\alpha=0.8575$ across the five subscales of the 15 -item short form, sufficient reliability was retained for the QABF-SF to be considered useful in clinical and research settings. It should be noted that, consistent with the original, the internal consistency of the QABF-SF as a whole was lower than the mean of the subscales. However, this was expected given that both measures are designed to tap five unrelated variables (Paclawskyj et al., 2000). Other reliability estimates of the QABF-SF showed that the short form also retained the high degree of both test-retest and interrater reliability of the original QABF.

Social validity data that were also gathered during the QABF-SF tryout showed that, overall, frontline staff liked the format of the shortened measure, seemed to think that the QABF-SF could provide useful clinical information, and be useful as part of a brief functional assessment of behavior in individuals with ID. Of the frontline staff questioned, 14 (9.9\%) did not like the format of the QABF-SF, 23 (16\%) did not think that the QABF-SF would provide the 
team with good information, and 17 (12\%) did not think that the QABF-SF could be useful as part of a brief functional assessment. Although these rejection rates were low, it was useful to determine possible reasons that staff did not like the QABF-SF. Regarding the QABF-SF, staff cited the time of day and shift that staff was asked to respond (i.e., morning vs. night), personal beliefs about individuals with ID, knowledge of behavioral assessment principles and techniques, and experience with the rating scale development process.

Given the often complex nature of maladaptive behavior exhibited by individuals with ID, the QABF-SF can be clinically useful in providing a starting point in the development of a behavioral support plan. Traditionally, one of the first steps when designing a behavioral intervention is to develop a hypothesis regarding the function that the behavior serves for the individual. The QABF-SF fulfills this role as an aid to clinicians in developing this hypothesis. However, the QABF-SF remains a clinical screening instrument and is it likely that second order assessments may be needed as indicated in confirming or disconfirming the possible function(s) of maladaptive behaviors that individuals with ID engage in. For example, if the function of a particular behavior such as physical aggression seems to be escape, it is critical to ask what the nature of the escape behavior is, in what context(s) it occurs, and what maintains the behavior. The answers to questions such as these will assist behavioral psychologists develop functionally equivalent replacement behaviors to teach the individual while examining more closely some of the environmental and systemic factors that may be helping to maintain the individual's behavior.

The current findings should be considered in light of several limitations. First, the samples consisted primarily of individuals diagnosed with severe to profound ID. Thus, the current analysis cannot determine whether or not the factor structure would differ among individuals with mild to moderate levels of ID. Second, the relatively small number of 
participants in the QABF-SF tryout limits the model reliability and thus results concerning the reliability of the QABF-SF should be interpreted with caution. Future investigations conducted with larger sample sizes are needed to replicate these results and expand upon the present findings. Third, it should be noted that the use of modification indices to guide the CFA analyses, specifically the LeGrange multiplier, increases the probability of these findings being influenced by chance. Fourth, the relative lack of other psychometrically sound measures of behavioral function also limits the conclusions regarding the construct validity of this measure.

The results of the current study generated a number of future directions for research and theoretical implications. While the present study is offered to show an example of how researchers are continually refining measurement procedures in an effort to better tailor our treatments, additional psychometric and normative data are needed before the QABF-SF should be used clinically. These investigations should include studies that further examine the construct, convergent, and discriminant validity of the QABF-SF. Other studies also need to be conducted to determine effects of shortening one measure in a battery affects responder compliance and the overall reliability and validity of the data collected. In addition, the validity and clinical utility of the QABF-SF should be further assessed by determining whether the five factor model structure of this measure is related to critical outcomes and improved quality of life indices such as improved behavioral functioning, coping skills, and social functioning. Finally, the sensitivity of the QABF-SF to the type of ID and severity of behavioral problems during the course of a prescribed treatment should be examined.

In summary, there is a growing need for a simple, reliable screening tool to help identify the function(s) of maladaptive behavior exhibited by individuals with intellectual disabilities. Literature on other methods of behavioral assessment, such as analog functional analysis, shows that the benefit of engaging in such an endeavor does not outweigh costs in time and resources. 
Direct observation, while a cornerstone of behavioral assessment, sometimes has limited applicability especially in situations where the behavior of concern is low in frequency. Thus, behavior rating scales have become one of the most widely used methods of collecting data about the possible function of an individual's behavior. The QABF-SF seems to be a tool that can be used as an alternative to the QABF when assessment time, staff knowledge, and limited resources must be maximized. 


\section{REFERENCES}

Allen, E.L. (1957). Techniques of attitude scale construction. New York: Appleton, Century and Crofts.

Aman, M.G, \& Singh, N.N. (1986). Aberrant Behavior Checklist (ABC). East Aurora, NY: Slosson Educational Publications, Inc.

American Psychiatric Association (2000). Diagnostic and statistical manual of mental disorders (4th ed., text revision). Washington, DC: Author.

Andrich, D. (1982). An index of person separation in latent trait theory, the traditional KR.20 index, and the Guttman scale response pattern. Education Research and Perspectives, 9 , 95-104.

Applegate, H., Matson, J.L., \& Cherry, K.E. (1999). An evaluation of functional variables affecting severe behavior problems in adults with mental retardation by using the Questions About Behavioral Function Scale (QABF). Research in Developmental Disabilities, 20, 229-237.

Arrindell, W., \& van der Ende, J. (1985). An empirical test of the utility of the observations-tovariables ratios in factor and component analysis. Applied Psychological Measurement, $\underline{9}, 165-178$.

Bandura, A. (1969). Principles of behavior modification. NY: Holt, Rinehart and Watson, Inc.

Balthazar, E. E., \& Stevens, H. A. (1975). The emotionally disturbed, mentally retarded: A historical and contemporary perspective. Englewood Cliff, NJ: Prentice-Hall.

Bentler, P.M. (1990) Comparative fit indices in structural models, Psychological Bulletin, 107, 238-246.

Bentler, P.M., \& Bonnett, D.G. (1980) Significance tests and goodness of fit in the analysis of covariance structures, Social and Behavioral Sciences, $19,16$.

Bentler, P.M., \& Kano, Y. (1990). An empirical-test of the utility of the observation-to-variables ratio in factor and components-analysis. Applied Psychological measurement, 9, 165-178.

Biasini, F. J., Grupe, L., Huffman, L., \& Bray, N. W. (1999). Mental retardation: A symptom and a syndrome. In S. D. Netherton \& $\leftarrow$ D. Holmes (Eds.) Child and adolescent psychological disorders: A comprehensive textbook (pp. 6-23). New York: Oxford University.

Blatt, B., \& Kaplan, F. (1966). Christmas in purgatory: A photographic essay on mental retardation. Boston: Allyn \& Bacon.

Bock, R.D., \& Jones, L.V. (1968). The measurement and prediction of judgment and choice. San Francisco: Holden-Day. 
Bollen, K.A. (1989). Structural equations with latent variables. New York: Wiley.

Boyle, G.J. (1991). Does item homogeneity indicate internal consistency or item redundancy in psychometric scales? Personality and Individual Differences, 12, 291-294.

Bowman, M.L. (1989). Testing individual differences in ancient China. American Psychologist, $\underline{44}, 576-578$.

Bryant, F.B., \& Yarnold, P.R. (1995). Principal-components analysis and exploratory and confirmatory factor analysis. In L.G. Grimm and P.R. Yarnold (Eds.). Reading and understanding multivariate statistics. Washington DC: American Psychological Association.

Byrne, B. (1998). Structural Equation Modeling with LISREL, PRELIS, and SIMPLIS: Basic applications and programs. NJ: Lawrence Erlbaum.

Carr, E.G. (1994). Emerging themes in the functional analysis of problem behavior. Journal of Applied Behavior Analysis, 27, 393-399.

Carr, E.G., \& Durand, V.M. (1985). Reducing behavior problems through functional communication training. Journal of Applied Behavior Analysis, 18, 111-126.

Cattell, R.B. (1950). Personality. New York: McGraw-Hill.

Child, D. (1990). The essentials of factor analysis ( $2^{\text {nd }}$ ed.). London: Cassel Educational Limited.

Cohen, R.J., \& Swerdlik, M.E. (1998). Psychological testing and assessment: An introduction to tests and measurement $\left(4^{\text {th }}\right.$ ed. $)$. London: Mayfield Publishing Company.

Committee on Classification of Feeble-Minded. (1910). Journal of Psycho-Asthenics, 15, 61-67.

Costello, A.B., \& Osborne, J.W. (2005). Best practices in exploratory factor analysis: Four recommendations for getting the most from your analysis. Practical Assessment, Research \& Evaluation, 10, 1-9.

Crocker, L.M., \& Algina, J. (1986). An introduction to classical and modern test theory. Belmont, CA: Wadsworth Group/Thomson Learning.

Cronbach, L.J. (1951). Coefficient alpha and the internal structure of tests. Psychometrika, 16, 297-334.

Derby, K.M., Wacker, D.P., Sasso, G., Steege, M., Northup, J., Cigrand, K., \& Asmus, J. (1992). Brief functional assessment techniques to evaluate aberrant behavior in an outpatient clinic: A summary of 79 cases. Journal of Applied Behavior Analysis, 25, 713-721.

Doll, E.A. (1953). The measurement of social competence: A manual for the Vineland Social Maturity Scale. Washington, DC: Educational Test Bureau. 
Doll, E.A. (1962). Trends and problems in the education of the mentally retarded: 1800-1940. American Journal of Mental Deficiency, 72, 175-183.

Dura, J. (1997). Expressive communicative ability, symptoms of mental illness and aggressive behavior. Journal of Clinical Psychology, 53, 307-318.

Durand, V.M. \& Carr, E.G. (1991). Functional communication training to reduce challenging behavior: Maintenance and application in new settings. Journal of Applied Behavior Analysis, 24, 251-264.

Durand, V.M., \& Carr, E.G. (1992). An analysis of maintenance following functional communication training. Journal of Applied Behavior Analysis, 25, 777-794.

Durand, V. M., \& Crimmins, D. B. (1988). Identifying variables maintaining self-injurious behavior. Journal of Autism and Developmental Disorders, 18, 99-117.

Embretson, S. \& Reise, S. (2000). Item response theory for psychologists. Mahwah, NJ: Erlbaum.

Fabrigar, L.R., Wegener, D.T., MacCallum, R.C., \& Strahan (1999). Evaluating the use of exploratory factor analysis in psychological research. Psychological Methods, 4, 272299.

Flanagan, D. P., Genshaft, J. L., \& Harrison, P. L. (Eds.). (1997). Contemporary intellectual assessment: Theories, tests, and issues. NY: Guilford.

Floyd, F.J., \& Widaman, K.F. (1995). Factor analysis in the development and refinement of clinical assessment instruments. Psychological Assessment, 7, 227-236.

Gable, R.A. (1996). A critical analysis of functional assessment: Issues for researchers and practitioners. Behavioral Disorders, 22, 36-40.

Galfo, A.J. (1985). Teaching degrees of freedom as a concept in inferential statistics: An elementary approach. School Science and Mathematics, 85, 240-247.

Good, I.J. (1973). What are degrees of freedom? American Statisticians, 27, 227-228.

Gorsuch, R.L. (1990). Common factor analysis versus component analysis: Some well and little known facts. Multivariate Behavioral Research, 25, 33-39.

Greenspan, S. (1999). What is meant by mental retardation? International Review of Psychiatry, 11, 6-18.

Grossman, H.J. (1973). Manual on terminology in mental retardation (1973 rev Ed.). Washington, DC: American Association on Mental Deficiency. 
Grossman, H.J. (1977). Manual on terminology in mental retardation (1977 rev Ed.)

Washington, DC: American Association on Mental Deficiency.

Haynes, S.N., \& O’Brien, W.H. (1990). Functional analysis in behavior therapy. Clinical Psychology Review, 10, 649-668.

Iwata, B.A. (1994). Functional analysis methodology: Some closing comments. Journal of Applied Behavior Analysis, 27, 413-418.

Iwata, B., Dorsey, M., Slifer, K., Bauman, K., \& Richman, G. (1982). Toward a functional analysis of self-injury. Analysis and Intervention in Developmental Disabilities, 2, 3-20.

Iwata, B.A., Pace, G., Kalsher, M., Cowdery, G., \& Cataldo, M. (1990). Experimental analysis and extinction of self-injurious escape behavior. Journal of Applied Behavior Analysis, 23, $11-27$.

Jaccard, J., \& Becker, M.A. (1990). Statistics for the behavioral sciences. (2 ${ }^{\text {nd }}$ Ed.). Belmont, CA: Wadsworth.

Joreskog, K. G., \& Sorbom, D. (1984). LISREL VI: Analysis of linear structural relationships by the method of maximum likelihood Chicago, IL: National Educational Resources.

Kass, R., \& Tinsley, H. (1979). Factor analysis. Journal of Leisure Research, 11, 120-138.

Kline, T.B. (2005). Psychological testing: A practical approach to design and evaluation. London: Sage Publications, Inc.

Kim, J.O., \& Mueller, C.W. (1978a). Introduction to factor analysis: What it is and how to do it. Newbury Park, NY: Sage.

Kim, J.O., \& Mueller, C.W. (1978b). Factor analysis: Statistical methods and practical issues. Newbury Park, NY: Sage.

Lawley, D.N. and Maxwell, A.E. (1971). Factor analysis as a statistical method. London: Butterworth and Co.

Likert, R. (1932). A technique for the measurement of attitudes. Archives of Psychology, 140, 553.

Lohman, D.F. (1989). Human intelligence: An introduction into advances in theory and research. Review of Educational Research, 59, 333-374.

Lord, F.M. (1959). Tests of the same lengths do have the same standard error of measurement. Educational and Psychological Measurement, 19, 233-239.

Lord, F.M. (1980). Applications of item response theory to practical testing problems. Mahwah, NJ: Erlbaum. 
Lord, F.M., \& Novik, M.R. (1968). Statistical theories of mental test scores. Reading, MA: Addison-Wesley.

Lowrey, M. \& Sovner, R. (1991). The functional significance of problem behavior: A key to effective treatment. Habilitative Mental Healthcare Newsletter, 10, 59-62.

Mathias, J. L., \& Nettlebeck, T. (1992). Validity of Greenspan's models of adaptive and social intelligence. Research in Developmental Disabilities, 13, 113-129.

Matson, J. L. (1995a). The Diagnostic Assessment for the Severely Handicapped-II. Baton Rouge, LA: Scientific Publishers Inc.

Matson, J.L. (1995b). The Matson Evaluation of Social Skills for Individuals with Severe Retardation (MESSIER). Baton Rouge, LA: Disability Consultants, LLC.

Matson, J. L., \& Bamburg, J. W. (1998). Reliability of the Assessment of Dual Diagnosis (ADD). Research in Developmental Disabilities, 19, 89-95.

Matson, J.L., \& Kuhn, D.E. (2001). Identifying feeding problems in mentally retarded persons: Development and reliability of the Screening Tool of fEeding Problems (STEP). Research in Developmental Disabilities, 21, 165-172.

Matson, J.L., Bamburg, J.W., Cherry, K.E., \& Paclawskyj, T.R. (1999). A validity study on the Questions About Behavioral Function (QABF) Scale: Predicting treatment success for self-injury, aggression, and stereotypies. Research in Developmental Disabilities, 20, 163 176.

Matson, J.L., Mayville, S.B., Kuhn, D.E, Sturmey, P., Laud, R.B., \& Cooper, C.L. (2001). The behavioral function of feeding problems as assessed by the Questions About Behavioral Function (QABF). Research in Developmental Disabilities, 26, 399-408.

Matson, J.L., Vollmer, T.R., Paclawskyj, T.R., Smiroldo, B.B., Applegate, H.R., \& Stallings, S. (1996). Questions About Behavioral Function (QABF): An instrument to assess functional properties of problem behaviors. Poster presented at the $22^{\text {nd }}$ Annual Convention of the Association for Behavior Analysis, San Francisco, CA.

Menolascino, F.J., Levitas, A., \& Greiner, C. (1986). The nature and types of mental illness in the mentally retarded. Psychopharmacology Bulletin, 22, 1060-1071.

Muliak, S.A. (1990). Blurring the distinctions between component analysis and common factor analysis. Multivariate Behavioral Research, 25, 53-59.

Nicholson, J., Konstantinidi, E., \& Furniss, F. (2006). On some psychometric properties of the Questions About Behavioral Function scale (QABF). Research in Developmental Disabilities, 27, 337-352. 
Nihira, K., Foster, R., Shellhaas, M., \& Leland, H. (1969). AAMD Adaptive Behavior Scale. Washington, DC: American Association on Mental Deficiency.

Nihira, K., Leland, H., \& Lambert, N. (1993). AAMR adaptive behavior scale residential and community (2nd edition): Examiner's manual. Austin, TX: PRO-ED.

O’Neill, R.E., Horner, R.J., Albin, R.W., Storey, K., \& Sprague, J.R. (1990). Functional analysis of problem behavior: A practical assessment guide. Sycamore, IL: Sycamore.

Paclawskyj, T.R., Matson, J.L., Rush, K.S., Smalls, Y., \& Vollmer, T.R. (2000). Questions About Behavioral Function (QABF): A behavioral checklist for functional assessment of aberrant behavior. Research in Developmental Disabilities, 21, 223-229.

Robertson, G.J. (1990). A practical model for test development. In C.R. Reynolds \& R.W. Kamphaus (Eds.). Handbook of psychological and educational assessment of children: Intelligence \& achievement (pp. 62-85). New York: Guilford.

Rojahn, J., Polster, L. M., Mulick, J. A., \& Wisniewski, J. J. (1989). Reliability of the Behavior Problems Inventory. Journal of the Multihandicapped Person, 2, 283-293.

Sheerenberger, R.C. (1983). A history of mental retardation. Baltimore: Brookes Publishing Co.

Singh, N.N., Matson, J.L., Lancioni, G.E., Singh, A.N., Adkins, A.D., McKeegan, G.F., \& Brown, S.W. (2006). Questions About Behavioral Function in Mental Illness (QABF-MI): A behavior checklist for functional assessment of maladaptive behavior exhibited by individuals with mental illness. Behavior Modification, 30, 739-751.

Skinner, B.F. (1953). Science and human behavior. NY: Macmillan.

Smith, R.G., Iwata, B.A., Vollmer, T.R., \& Zarcone, J.R. (1993). Experimental analysis and treatment of multiply controlled self-injury. Journal of Applied Behavior Analysis, 26, $183-196$.

Snook, S.C., \& Gorsuch, R.L. (1989). Principal component analysis versus common factor analysis: A Monte Carlo study. Psychological Bulletin, 106, 148-154.

Sparrow, S., Balla, D., \& Cicchetti, D. (1984). Vineland Adaptive Behavior Scales. Circle Pines, MN: American Guidance Service.

Sternberg, R.J. \& Grigorenko, E.L. (1997). Are cognitive styles still in style? American Psychologist, 52, 700-712.

Steiger, J.H. \& Lind, J.C. (1980). Statistically-based tests for the number of common factors. Paper presented at the annual Spring Meeting of the Psychometric Society in Iowa City, IA. 
Sturmey, P. (1995). Analog baselines: A critical review of the methodology. Research in Developmental Disabilities, 12, 269-284.

Taylor, J.C., Ekadahl, M.M., Romancyzk, R.G., \& Miller, M.L. (1994). Escape behavior in task situations: Task versus social antecedents. Journal of Autism and Developmental Disabilities, 24, 331-344.

Thompson, B. (2004). Exploratory and confirmatory factor analysis: Understanding concepts and applications. Washington DC: American Psychological Association.

Toothaker, L.E., \& Miller, L. (1996). Introductory statistics for the behavioral sciences. (2 ${ }^{\text {nd }}$ Ed.). Pacific Grove, CA: Brooks/Cole.

Thurston, L.L. (1959). The measurement of values. Chicago: University of Chicago.

Van der Linden, W.J. \& Hambleton, R.K. (Eds.) (1997). Handbook of modern item response theory. NY: Springer.

Velicer, W.F., \& Jackson, D.N. (1990). Component analysis versus common factor analysis: Some further observations. Multivariate Behavioral Research, 25, 231-25l.

Vollmer, T.R, \& Matson, J.L. (1995). User's guide: Questions About Behavioral Function $\underline{(\mathrm{QABF})}$. Baton Rouge, LA: Scientific Publishers, Inc.

Wacker, D.P., Steege, M.W., Northup, J., Sasso, G., Berg, W., Reimers, T., Cooper, L., Cigrand, K., \& Donn, L. (1990). A component analysis of functional communication training across three topographies of severe behavior problems. Journal of Applied Behavior Analysis, 23, 417-429.

Weeks, M., \& Gaylord-Ross, R. (1981). Task difficulty and aberrant behavior in severely handicapped students. Journal of Applied Behavior Analysis, 14, 449-463.

Yepsen, L. (1941). Defining mental deficiency. American Journal of Mental Deficiency, 46, 200205. 


\section{VITA}

Ashvind Nand Singh was born in Auckland, New Zealand, and moved to the United

States at the age of 11. Ashvind went to the Virginia Commonwealth University and received a Bachelor of Science with Honors in psychology in 1999. He is currently enrolled in the clinical psychology doctoral program at Louisiana State University, working under Dr. Johnny L. Matson. His research and clinical areas of interest include forensic psychology, mindfulness, psychopharmacology, and neurocognition. 